\title{
Data Report for the Main Line of the PSINE Seismic Survey Across the San Andreas Fault and SAFOD Site Near Parkfield, California by
}

R. D. Catchings, M. R. Goldman, M. J. Rymer, G. Gandhok, and G. S. Fuis

Open-File Report 03-84

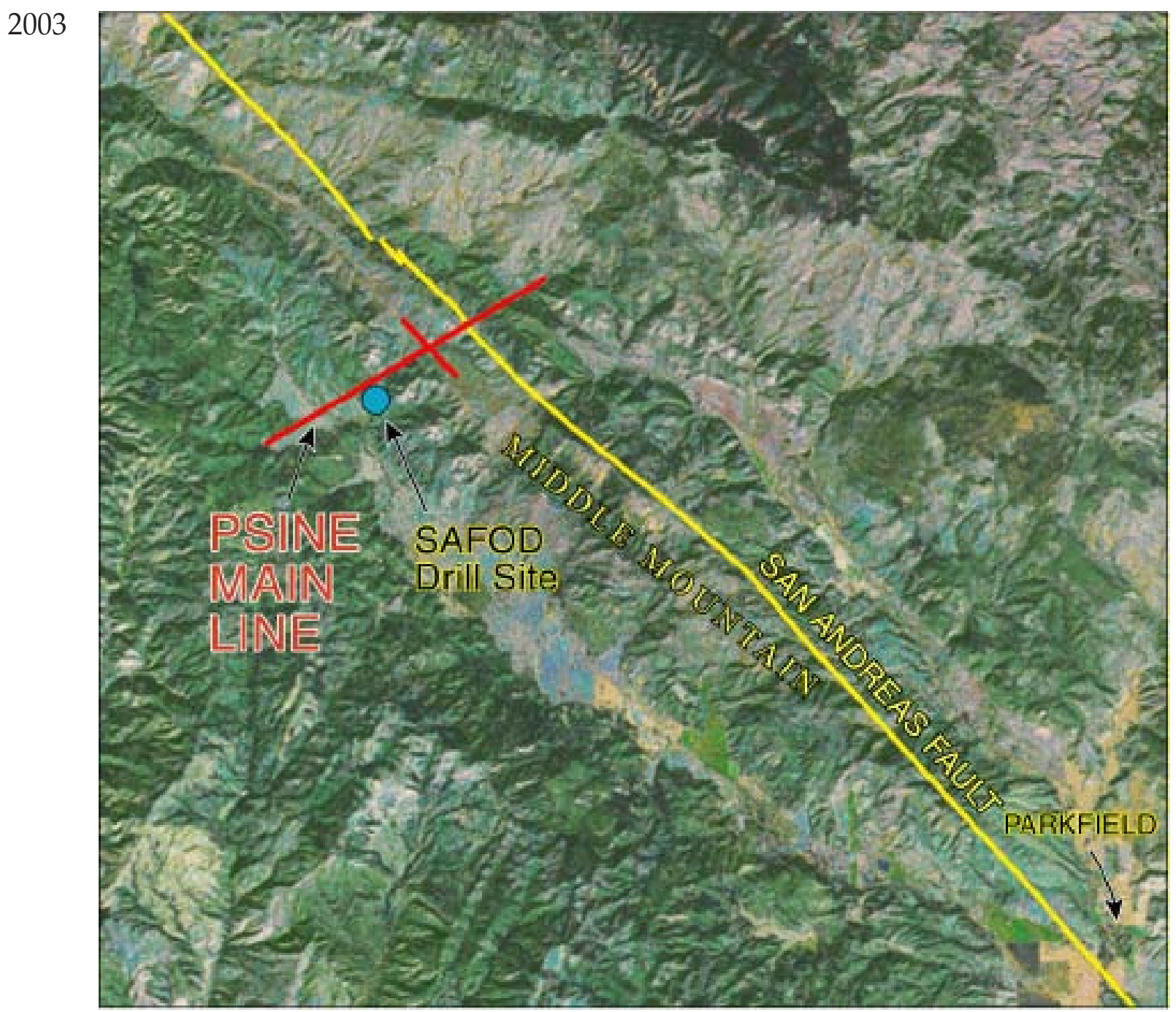

This report is preliminary and has not been reviewed for conformity with U.S. Geological Survey editorial standards or with the North American Stratigraphic Code. Any use of product names is for descriptive purpose only and does not imply endorsement by the U.S. Government.

\section{U.S. DEPARTMENT OF THE INTERIOR} U.S. GEOLOGICAL SURVEY

Menlo Park, California 


\section{Introduction}

In October and November 1998, the U.S. Geological Survey, Virginia Polytechnic Institute, Stanford University, and Geometrics Corporation jointly acquired a highresolution seismic reflection/refraction profile across the San Andreas Fault Zone (SAFZ) and the proposed San-Andreas-Fault-Observatory-at-Depth (SAFOD) drill site located near Parkfield, California (Fig. 1a). We refer to this seismic survey as the Parkfield Seismic Imaging-Ninety Eight (PSINE) survey, the highest-resolution seismic survey yet completed across the SAF in central California. This report, which presents acquisition parameters and example shot gathers for the PSINE seismic survey, is intended to provide necessary information for those who wish to use the PSINE data for research.

The PSINE survey is part of the proposed SAFOD investigation: a proposed study to investigate physical and chemical properties and processes that control deformation and earthquake generation within the SAF zone by drilling into the active trace of the fault (Zoback et al., 1998; www.earthscope.org). Geological and geophysical investigations are planned prior to, during, and after completion of the borehole. The PSINE survey, which was one of the pre-drilling investigations, was designed to determine the subsurface stratigraphic and structural relations in the vicinity of the proposed drill site, to better locate the target fault zone within the subsurface, and to image obstacles that may impede drilling. Data acquisition parameters, experiment setup, and parameters needed to work with the PSINE data are provided in this report. The PSINE data provide useful reflective images (Catchings et al., in prep; Rymer et al., in prep; Hole et al.,) and velocity data (Catchings et al., 2002) in the vicinity of the SAFOD drill site and the San Andreas fault.

\section{Local Geology and Tectonics}

The San Andreas fault is a right-lateral, strike-slip fault that extends approximately $1100 \mathrm{~km}$ from near the U.S.-Mexican border to northwest of Cape Mendicino, California, and it forms the boundary between the Pacific plate to the southwest and the North American plate to the northeast (Fig. 1a). The town of Parkfield lies near the central segment of the SAF and has been considered an important area for study of earthquakes over the past 20 years. Historically in the Parkfield area, moderate magnitude (M 5.5 to 6) earthquakes have occurred on the SAF on average every few decades (Bakun and McEvilly, 1984; Bakun and Lindh, 1985). However, small to moderate earthquakes ( $\mathrm{M} \sim 4$ or less) occur more frequently (Nadeau and McEvilly, 1997). In the Parkfield area, the SAF has a long history of strike-slip movement within the present surface fault zone and along nearby sub-parallel faults (Dickinson, 1966; Sims, 1992; Rymer et al., in prep.). Although there are numerous mapped faults in the Parkfield area, the most recently active surface trace extends along the length of Middle 
Mountain, a roughly symmetrical ridge about $15 \mathrm{~km}$ long and about $3.2 \mathrm{~km}$ wide (Fig. 1). Within our study area, the surface trace of the SAF lies east of the crest of Middle Mountain (Dickinson, 1966; Rymer et al., in prep.).

\section{PSINE Data Acquisition}

The PSINE survey consisted of three separate seismic surveys: (1) an approximately 5-km-long seismic reflection and refraction survey across the SAF (main line), (2) a 1-km-long seismic reflection and refraction survey parallel to the SAF, and (3) a 3-D reflection and refraction survey across the active surface trace of the SAF (Fig. 1b). This report describes data acquisition parameters only along the main profile of the PSINE survey.

The main PSINE profile was approximately centered on the proposed SAFOD drill site and was oriented $\sim \mathrm{N} 55^{\circ} \mathrm{E}$, which is slightly less than perpendicular to the active trace of the SAF $\left(\sim \mathrm{N} 45^{\circ} \mathrm{W}\right)$ (Fig. 1b). Approximately $3.5 \mathrm{~km}$ of the seismic profile was on the southwest side of the surface trace of the SAF, with the remaining $\sim 1.5 \mathrm{~km}$ of the profile on the northeast side. The main seismic profile consisted of an $\sim 5-\mathrm{km}$-long, cabled recording array with 13 linked Geometrics ${ }^{\mathrm{TM}}$ multi-channel seismographs (Fig. 1c). Of the 13 multi-channel seismographs ( 840 channels) used, one seismograph was a 120-channel unit, and the remaining 12 seismographs were 60 -channel units. Figures $1 \mathrm{~b}$ and $\mathrm{c}$ show the setup for the seven recording sites along the main profile. With the exception of site 1, there were two 60-channel seismographs at each site, with 60 channels deployed to the northeast and 60 channels deployed to the southwest of the site. At site 1, a single 120-channel seismograph was deployed with a total of 840 channels along the main profile. Sensors consisted of a combination of $40-\mathrm{Hz}, 28-\mathrm{Hz}$, and $10-\mathrm{Hz}$ Mark Products ${ }^{\mathrm{TM}}$ geophones, spaced at 5-m intervals over the seismic array, except near the southwestern $1.2 \mathrm{~km}$ and the northeastern $0.6 \mathrm{~km}$ of the profile (see Table 2), where the geophone spacing was $10 \mathrm{~m}$. The $10-\mathrm{Hz}$ geophones were deployed on the southwestern $(300 \mathrm{~m})$ and northeastern $(600-\mathrm{m})$ ends of the profile, and the $40-\mathrm{Hz}$ and $28-\mathrm{Hz}$ geophones were used in the central part of the profile.

Shot points were spaced at a constant 10-m interval over the entire array, and shots were co-located with the geophones (1 m lateral offset). Most shots consisted of 1or 2-lb explosions of ammonium nitrate in $\sim 3$-m-deep shot holes, but where it was not feasible to use explosive shots, we used 400-grain, Betsy-Seisgun ${ }^{\mathrm{TM}}$ blanks in $\sim 0.5$-mdeep holes.

Timing for the explosive shots was accomplished with USGS shooting systems that were synchronized with USGS master clocks. The USGS master clocks were used to trigger the seismographs approximately $50 \mathrm{~ms}$ before the explosion. Seismograph pairs at each recording site (Fig. 1c) were connected to a single master clock to maintain exact timing between the pairs. The most distant two channels at each site were also co-located 
with those of adjacent sites, as a secondary check on timing between sites. Timing for Betsy-Seisgun ${ }^{\mathrm{TM}}$ sources was accomplished by connecting all seismographs with a series of cables that were also attached to the Betsy Seisgun. The hammer used to fire the shots formed a closed circuit with the seismographs, resulting in an electrical signal being transmitted to the seismograph when the hammer made contact with the seisgun.

Acquisition and recording parameters for the main line of the PSINE survey are presented in Tables 1a and 1b. Data were recorded without acquisition filters for $5 \mathrm{~s}$ at a 0.5-ms sampling rate and stored on the hard disk of the seismograph during field acquisition. After recording each day, the data were retrieved from the hard disk of the seismographs and downloaded to 4-mm DAT tapes for permanent storage.

\section{Shot-Point and Receiver Locations}

To properly account for variations in geometry, each shot point and geophone location was surveyed using an electronic distance meter (EDM) or a Global Positioning System (GPS) with theoretical accuracies of a few centimeters. The geometry data for the main profile of the PSINE survey are presented in Appendix A and are shown graphically in figures 2-5. Geophone elevations varied by about $120 \mathrm{~m}$ along the profile (Fig. 2), with the highest elevation occurring at Middle Mountain near meter 3329 of the survey and the lowest elevation occurring near the western part of the seismic profile, near meter 1011. The array of geophones varied from a straight line (that connects the first and 220th geophone) by less than $40 \mathrm{~m}$ along the entire length of the 4893-m-long array (Fig. 3). Variations in shot point elevations were less than $120 \mathrm{~m}$ (Fig. 4), and the geophone locations varied from a linear array by no more than $40 \mathrm{~m}$ along the profile (Fig. 5).

\section{Theoretical Fold}

PSINE survey utilized a stationary recording array, with all channels active for all shots. This setup resulted in linearly varying fold along the seismic profile (Fig. 6). The maximum fold (466) occurs near the center of the seismic profile, and the minimum fold (2) occurs near the ends of the profile. For seismic reflection stacking purposes, the deeper structure should be best resolved near the central part (highest fold) of the seismic profile. However, the $\sim 1-\mathrm{kg}$ shots were energetic enough to yield reflections from about $5 \mathrm{~km}$ depth along the entire seismic profile (Catchings et al., in prep).

\section{Data Processing}

We minimally processed the shot gathers presented in this report to provide a more complete and useful data set. Because data from13 separate seismographs were 
combined to form the shot gathers, data from each seismograph was timed with adjacent seismographs using the USGS master clocks. Afterward, timing was checked using autocorrelation of seismic traces from co-located channels of adjacent seismographs. After individual shot gathers were assembled, we used trace editing to remove noisy or dead traces. For display purposes in the report, we used minimum phase bandpass filtering of 10-20-200-400 to help compensate for differing types of geophones along the seismic profile. However, the data available to researchers are unfiltered, with no acquisition or processing filters used on those data.

The geometrical setup of the PSINE survey generated seismograms for every $5 \mathrm{~m}$ of the profile, except near the ends, where the geophones were spaced every $10 \mathrm{~m}$. To make the seismogram spacing uniform along the entire array, we used trace in-filling near the ends of the seismic profile, whereby, the signal from adjacent (10-m-spaced) geophones were averaged to form a composite seismogram, and the resulting seismogram was inserted at one-half the distance between those stations. Because of the close spacing of recording channels $(10 \mathrm{~m})$ relative to the wavelength of the seismic signal, this technique worked well in providing an uniformly spaced shotgather.

Because of the large cumulative volume of data for the PSINE survey, we segmented the data into smaller volumes for ease in handling the data with the PROMAX processing package. For example, data labeled as series 1000 correspond to shot gathers between meters 0 and 531 of the seismic profile (see Table 1 and Fig. 1c). Shot gathers are labeled by FFID \# (Field File Identification Number). To determine the location of specific FFIDs, please see Fig. 1c and Table 3.

\section{Seismic Data}

We present representative shot gathers from the seismic profile (figures 7-11). Due to relatively low cultural noise in the area, signal-to-noise ratios were high, and most $\sim 1-\mathrm{kg}$ explosive sources were sufficient to propagate across the entire array. The data are available in SEGY format as shot gathers.

However, to fully understand the structure of the crust along the PSINE profile, more intensive processing of the data is required. To date, some of the data have been processed and the structure of the crust has been previously reported. First-arrival refractions were used to develop 2-D velocity models along the main line of the PSINE survey (Catchings et al., 2002), and near-vertical reflection images have been developed by stacking the shot gathers (Catchings, et al, in prep; Rymer et al., in prep). Steeply dipping reflections and diffractions have been migrated to develop an image of nearvertical structures in the crust (Hole et al., 2001). 


\section{Data Availability}

Data from the main line of the PSINE survey are available by contacting R. D. Catchings at the address listed on the front of this report or by contacting the IRISPASSCAL data center.

\section{Acknowledgements}

Funding for this seismic investigation was provided by the U.S. Geological Survey's Venture Capital Fund, the USGS-Menlo Park High-Resolution Seismic Imaging Group, and the USGS Western Earthquake Hazards Team. We thank Mr. Satoru Ohyo of Oyo Corporation and Geometrics Corporation for generous help in providing seismic equipment used in this study. We thank Mark Zoback, Steve Hickman, and William Ellsworth for providing logistical and informational assistance. We thank the Univ. of Idaho, the Univ. of South Carolina, the Univ. of California-Santa Cruz, the Virginia Polytechnic Institute, the USGS-Central Earthquake Hazards Team, and the USGSCoastal and Marine Geology Team for providing equipment. We thank Katherine Brown, Jean Chery, Joe Colgan, Coyn Criley, Ed Criley, Jeff Dingler, Andy Gallardo, Joe Grow, Gary Fuis, Balz Grollimund, Elba Horta, James Kessler, Grey Jensen, Heidrun Lelgemann, Mark Lemon, Janice Murphy, Keith Rice, Jose Rodriguez, John Towend, John VanShaack, Kristoffer Walker, Glen West, and Angie Williams for field assistance. We especially thank Kevin Kester for land access and assistance. We thank Shane Detweiler for reviewing the manuscript. 


\section{Figures}

Figure 1a. Location of the study area and the PSINE main profile of this report (heavy solid line). Faults from Jennings (1994).

Figure 1b. Layout of the PSINE profile relative to the San Andreas Fault Zone (SAFZ) and the San Andreas Fault Observatory at Depth (SAFOD) drill site. Numbers 1-9 refer to the site setup locations. Also shown are a cross line and a 3D array that were also recorded but not included in this report. Smaller numbers 001-936 along the profile refer to the channel (geophone) numbers. Base from Stockdale Mountain 7.5-minute quadrangle.

Figure 1c. Topographic profile along the PSINE seismic profile with site setup and data grouping information. Site setup \# (1-7) refers to the physical location of the recording sites (shown as black dots). Two 60-channel seismographs were located at all recording sites (except site 1), with 60 channels deployed to the northeast and 60 channels deployed to the southwest. At site 1, a single 120-channel seismograph was deployed. The dashed lines denote the limits of geophone deployments for each recording site. Shot gathers were subdivided into data series ranging from 1000 to 11000 for ease of data processing. The relative geographic locations of the data series (see Table 3) are shown. SAF refers to the mapped surface trace of the San Andreas fault. SP \# refers to shot-point locations (Field File ID Numbers) for shot gathers shown in figures 7-11.

Figure 2. Geophone elevation along PSINE seismic profile. Elevation is relative to the topographically lowest shot point along the profile.

Figure 3. Variation of geophones from a line that connects the first and $220^{\text {th }}$ geophone along the profile.

Figure 4. Shotpoint elevation along PSINE seismic profile. Elevation is relative to the topographically lowest shot point along the profile.

Figure 5. Variation of geophones from a line connecting the first and $110^{\text {th }}$ geophone along the profile.

Figure 6. Fold as a function of distance along the PSINE seismic profile.

Figure 7. Example shot gather with the shot point FFID 1010 located near the southwestern end of the PSINE profile. The horizontal axis shows the channel number of 
the recording array. The vertical axis is time in milliseconds. SAF refers to the surface trace of the San Andreas fault.

Figure 8. Example shot gather with the shot point FFID 1102 located near the southwest central part of the PSINE profile. The horizontal axis shows the channel number of the recording array. The vertical axis is time in milliseconds. SAF refers to the surface trace of the San Andreas fault.

Figure 9. Example shot gather with the shot point FFID 1224 located near the central part of the PSINE profile. The horizontal axis shows the channel number of the recording array. The vertical axis is time in milliseconds. SAF refers to the surface trace of the San Andreas fault.

Figure 10. Example shot gather with the shot point FFID 1333 located near the north central part of the PSINE profile. The horizontal axis shows the channel number of the recording array. The vertical axis is time in milliseconds. SAF refers to the surface trace of the San Andreas fault.

Figure 11. Example shot gather with the shot point FFID 1426 located near the northeastern part of the PSINE profile. The horizontal axis shows the channel number of the recording array. The vertical axis is time in milliseconds. SAF refers to the surface trace of the San Andreas fault. 


\section{References}

Bakun, W. H., and T. V. McEvilly (1984). Recurrence models and Parkfield, California, earthquake, J. Geophys. Res. 89, 3051-3058.

Bakun, W. H., and A. G. Lindh (1985). The Parkfield, California, earthquake prediction experiment, Science 229, 619-624.

Catchings, R. D., M. J. Rymer, M. R. Goldman, J.A. Hole, R. Huggins, and C. Lippus (2002). High-resolution seismic velocities and shallow structure of the San Andreas Fault Zone at Middle Mountain, Parkfield, California, Bull. Seis. Soc. Am. 92, 2493-2503.

Catchings, R. D., M. J. Rymer, and M. R. Goldman (in prep). High-resolution seismic reflection images of active strands of the San Andreas fault zone near Parkfield, California.

Dickinson, W. R. (1966). Structural relationships of San Andreas fault system, Cholame Valley, and Castle Mountain Range, California, Geol. Soc Am. Bull. 77, 707-726.

Hole, J. A., R. D. Catchings, K.C. St. Clair, M. R. Rymer, D.A. Okaya, and B.J. Carney (2001). Steep-dip seismic imaging of the shallow San Andreas Fault near Parkfield, Science, 294, 1513-1515.

Jennings, C. W. (Compiler). Fault activity map of California and adjacent areas, Calif. Dept. Conserv. Div. Mines Geol., Geologic Data Map Series No. 6, scale $1: 750,000$.

Nadeau, R. M., and T. V. McEvilly (1997). Seismological studies at Parkfield VI: Moment release rates and estimates of source parameters for repeating small eaerthquakes, Bull. Seis. Soc. Am. 88, 790-814.

Rymer, M.J. 1981, Geologic map along a 12-kilometer segment of the San Andreas fault zone, southern Diablo Range, California: U.S. Geol. Surv. Open-File Report 81-1173, scale $1: 12,000$.

Rymer, M. J., R. D. Catchings, M. R. Goldman, G. S. Fuis, R. Huggins, C. Lippus, and J. Hole (in prep). Shallow structure of the San Andreas fault zone near Parkfield, central California, as inferred from seismic reflection profiling and surface geology, Bull. Seis. Soc. Am. 
Sims, J.D., (1992). Chronology of displacement on the San Andreas fault in central California; evidence from reversed positions of exotic rock bodies near Parkfield, California, in The San Andreas Fault System; Displacement, Palinspastic Reconstruction, and Geologic Evolution, edited by R.E. Powell, R.J. Weldon, II, and J.C. Matti, Geol. Soc. Am. Mem. 178, 231-256.

Zoback, M. D., S. H. Hickman, and W. L Ellsworth (1998). Scientific drilling into the San Andreas fault at Parkfield, California: Project overview and operational plan, written manuscript proposal submitted to National Science Foundation, 146 pp; www.earthscope.org. 
Table 1a. Acquisition parameters for the PSINE main seismic profile. Distance is relative to the first shot point.

\begin{tabular}{|l|l|l|l|l|l|l|}
\hline Profile \# & Orientation & $\begin{array}{l}\text { Length of } \\
\text { geophone } \\
\text { Profile }(\mathrm{m})\end{array}$ & $\begin{array}{l}\text { Length of } \\
\text { shot Point } \\
\text { Profile }(\mathrm{m})\end{array}$ & $\begin{array}{l}\text { No. of } \\
\text { shots }\end{array}$ & $\begin{array}{l}\text { No. of } \\
\text { CDPs }\end{array}$ & $\begin{array}{l}\text { Maximum } \\
\text { fold }\end{array}$ \\
\hline $\begin{array}{l}\text { Parkfield } \\
\text { Main Line }\end{array}$ & SW-NE & 4893.75 & 4843.25 & 466 & 4868.5 & 466 \\
\hline
\end{tabular}

Table 1b. Acquisition and recording parameters for the PSINE main seismic profile.

\begin{tabular}{|l|l|l|l|}
\hline Sampling rate & Recording time & Filters & Pretrigger \\
\hline $0.5 \mathrm{~ms}$ & $5 \mathrm{sec}$ & out & $50 \mathrm{~ms}$ \\
\hline
\end{tabular}

Table 2. Site set up for the PSINE main line

\begin{tabular}{|c|c|c|c|}
\hline $\begin{array}{l}\text { Distance along } \\
\text { profile }\end{array}$ & Geophone type & Site setup \# & Geophone spacing \\
\hline $0-300 \mathrm{~m}$ & $10 \mathrm{~Hz}$ & 1 & $10 \mathrm{~m}$ \\
\hline $300 \mathrm{~m}-1.2 \mathrm{~km}$ & $40 \mathrm{~Hz}$ & 1 & $10 \mathrm{~m}$ \\
\hline $1.2 \mathrm{~km}-1.8 \mathrm{~km}$ & $40 \mathrm{~Hz}$ & 2 & $5 \mathrm{~m}$ \\
\hline $1.8 \mathrm{~km}-2.4 \mathrm{~km}$ & $40 \mathrm{~Hz}$ & 3 & $5 \mathrm{~m}$ \\
\hline $2.4 \mathrm{~km}-3.0 \mathrm{~km}$ & $40 \mathrm{~Hz}$ & 4 & $5 \mathrm{~m}$ \\
\hline $3.0 \mathrm{~km}-3.6 \mathrm{~km}$ & $40 \mathrm{~Hz}$ & 5 & $5 \mathrm{~m}$ \\
\hline $3.6 \mathrm{~km}-4.2 \mathrm{~km}$ & $40 \mathrm{~Hz}$ & 6 & $5 \mathrm{~m}$ \\
\hline $4.2 \mathrm{~km}-4.5 \mathrm{~km}$ & $28 \mathrm{~Hz}$ & 7 & $5 \mathrm{~m}$ \\
\hline $4.5 \mathrm{~km}-4.8 \mathrm{~km}$ & $10 \mathrm{~Hz}$ & 7 & $10 \mathrm{~m}$ \\
\hline
\end{tabular}

Table 3. Array set up for PSINE main seismic profile by data series, FFID \#, and distance.

\begin{tabular}{|c|c|c|}
\hline Series & FFID & Distance (meters) \\
\hline 1000 & $1001-1052$ & $0-530.8$ \\
\hline 2000 & $1053-1092$ & $540.66-960.17$ \\
\hline 3000 & $1093-1128$ & $970.66-1338.24$ \\
\hline
\end{tabular}




\begin{tabular}{|c|c|c|}
\hline $4-5000$ & $1129-1198$ & $1348.35-2064.33$ \\
\hline 6000 & $1199-1262$ & $2074.92-2775.52$ \\
\hline 7000 & $1263-1308$ & $2794.24-3238.51$ \\
\hline $9000 \mathrm{~A}$ & $1309-1359$ & $3247.57-3747.88$ \\
\hline $9000 \mathrm{~B}$ & $1360-1405$ & $3757.99-4238.44$ \\
\hline $10-11000$ & $1406-1466$ & $4248.52-4843.25$ \\
\hline
\end{tabular}




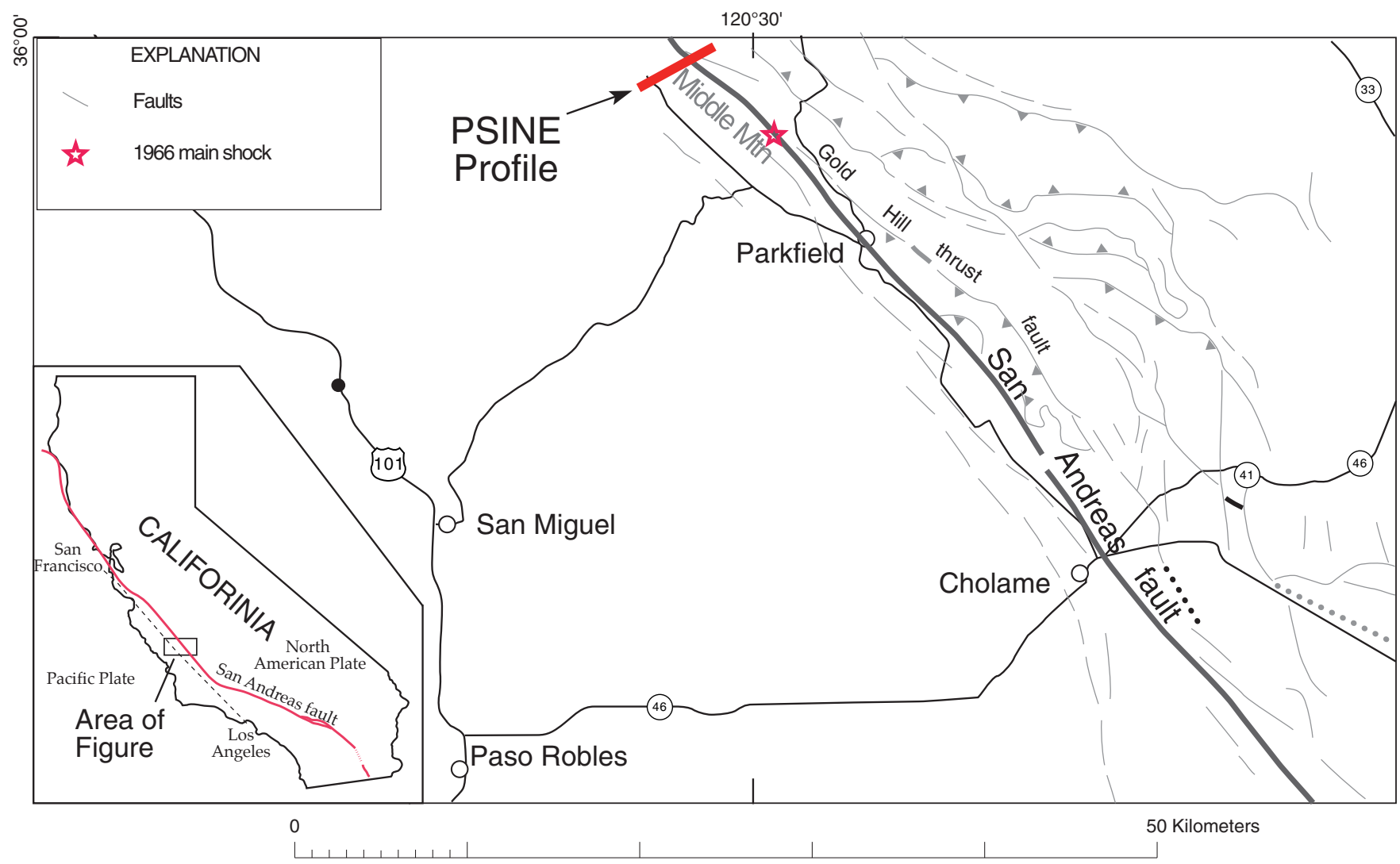

Fig 1a. 
$120^{\circ} 32^{\prime} 30^{\prime \prime}$

$36^{\circ} 00^{\prime}$

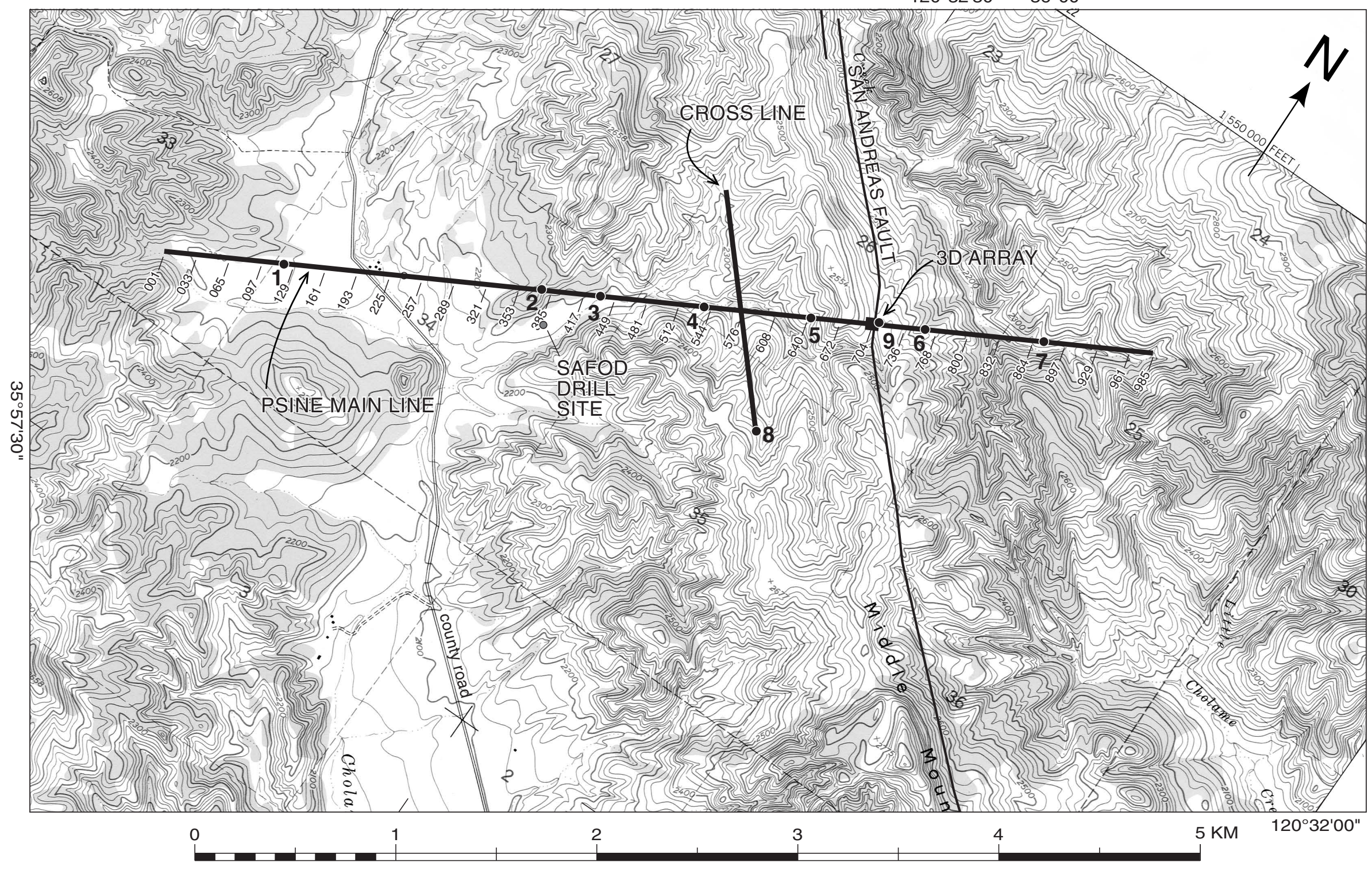


Southwest

Northeast

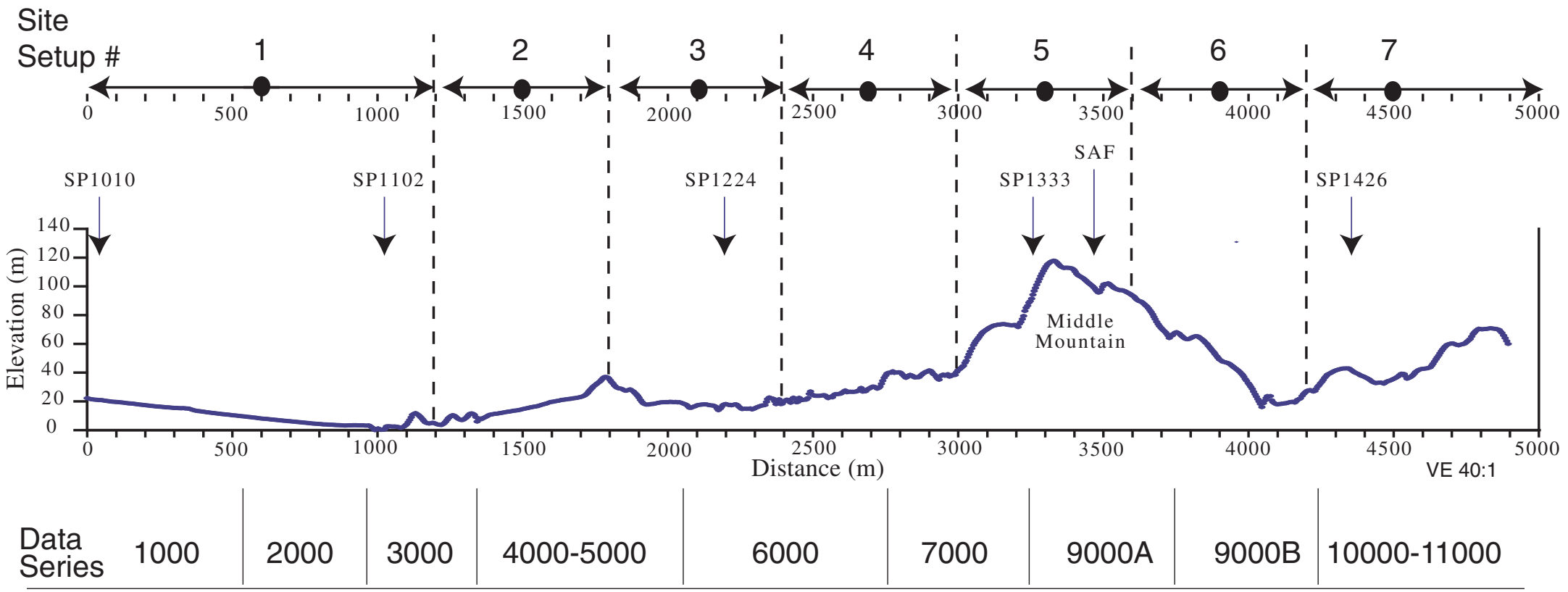

Fig. 1c. 


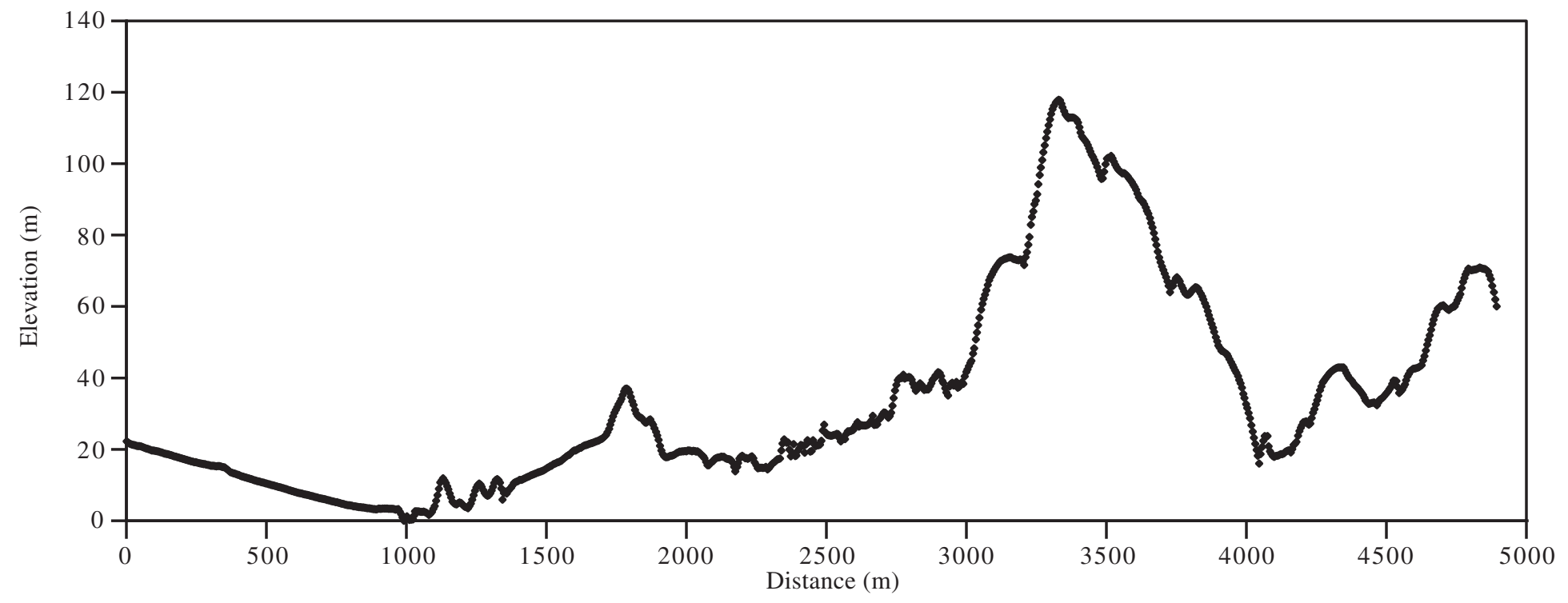

Fig. 2.

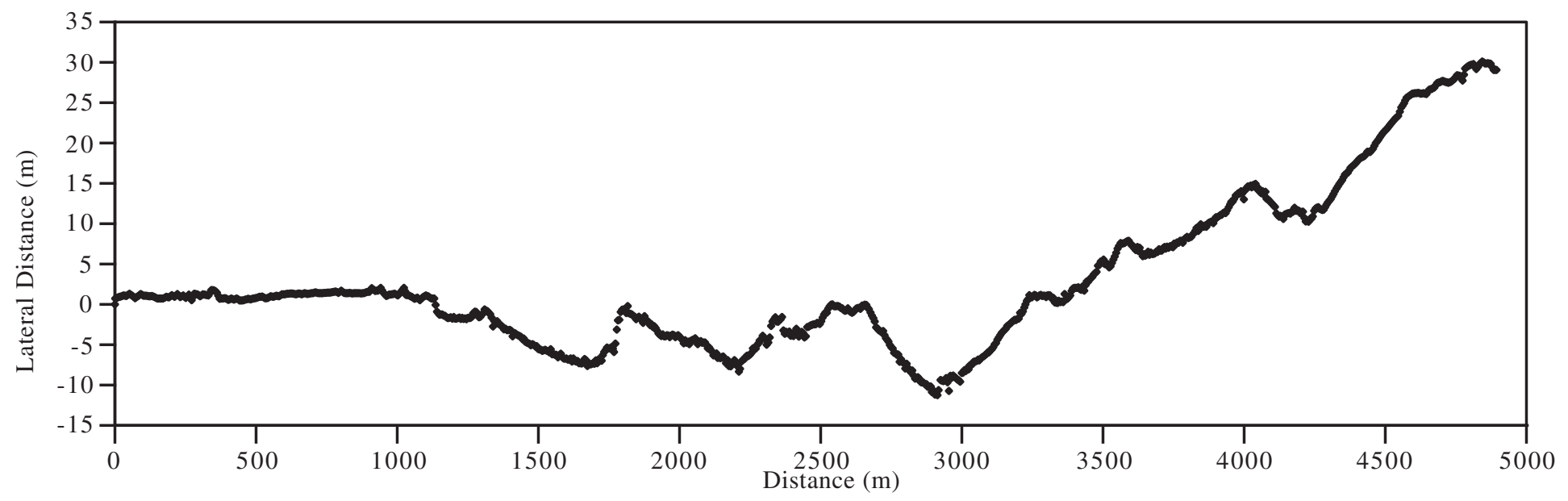

Fig. 3. 


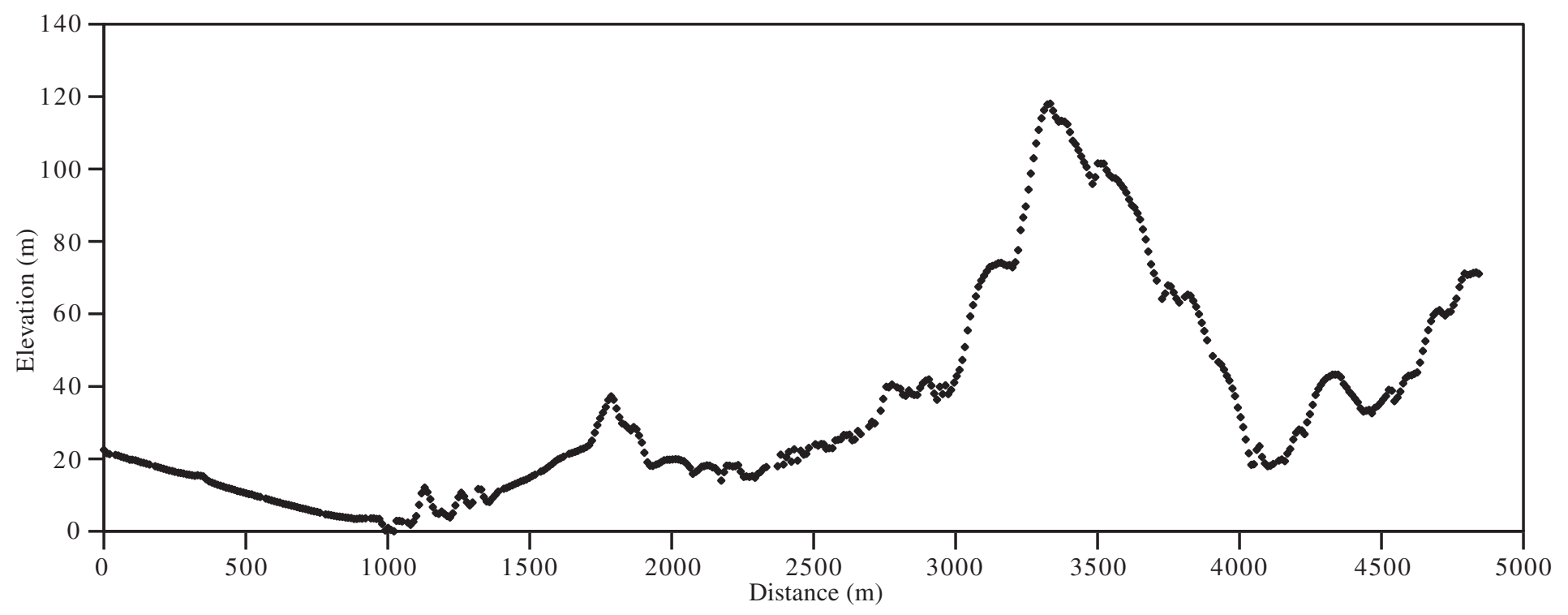

Fig. 4.

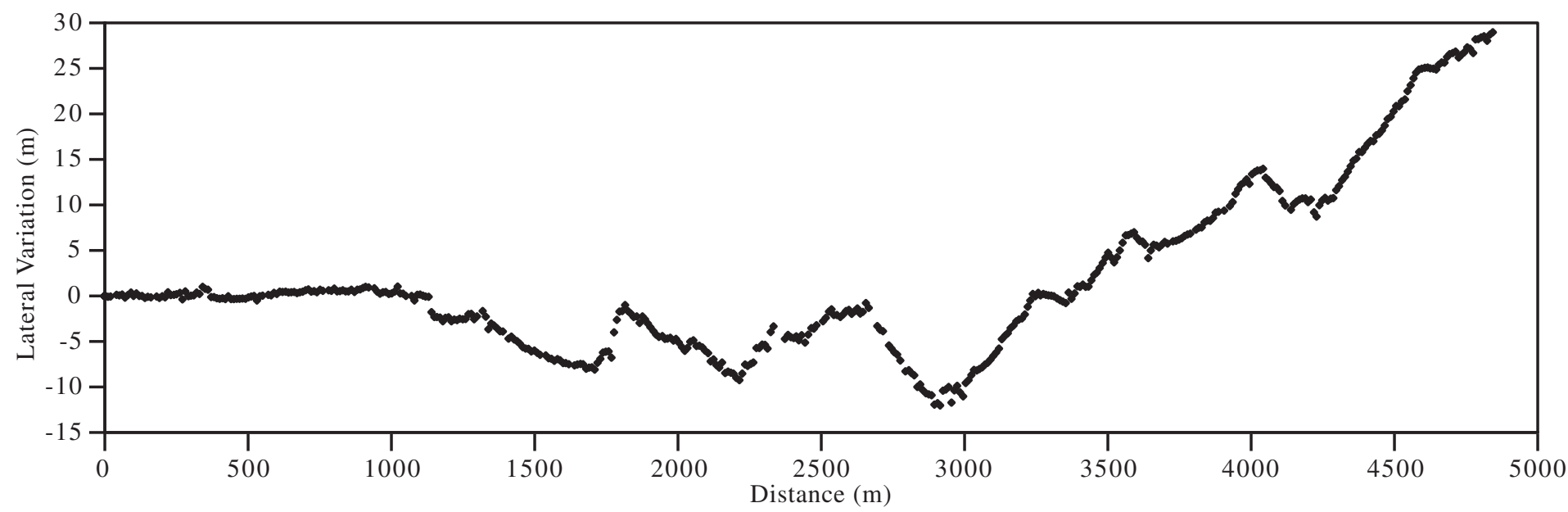

Fig. 5. 


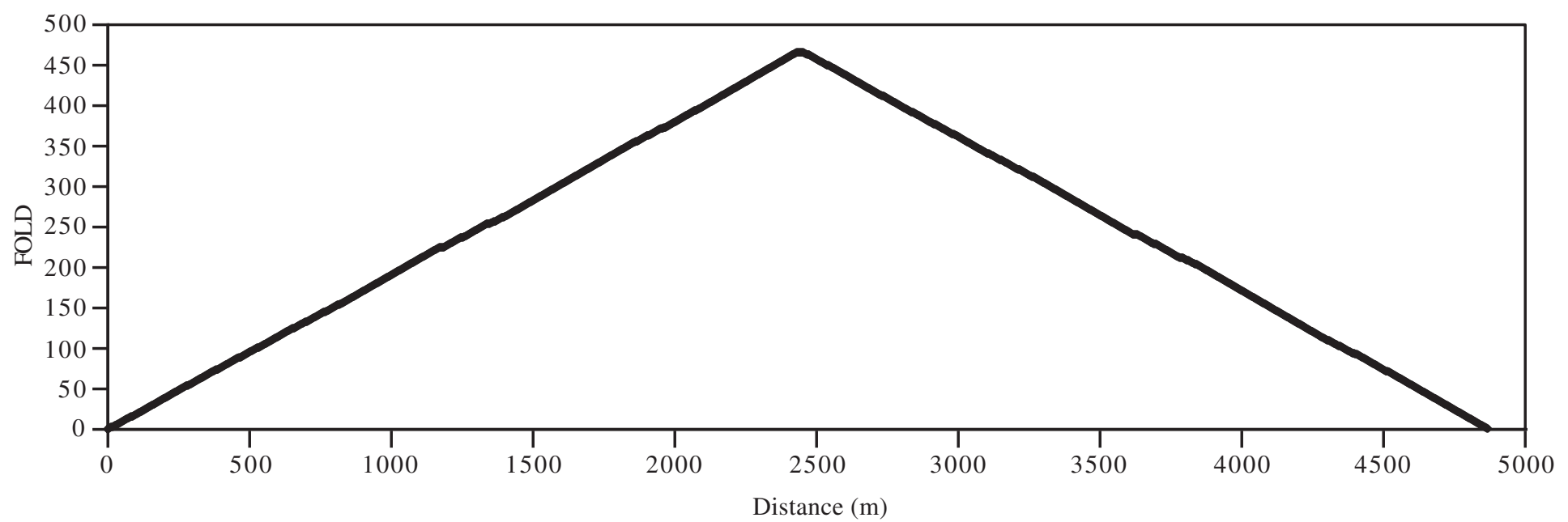

Fig. 6. 


\section{Parkfield - Line 1 FFID 1010}

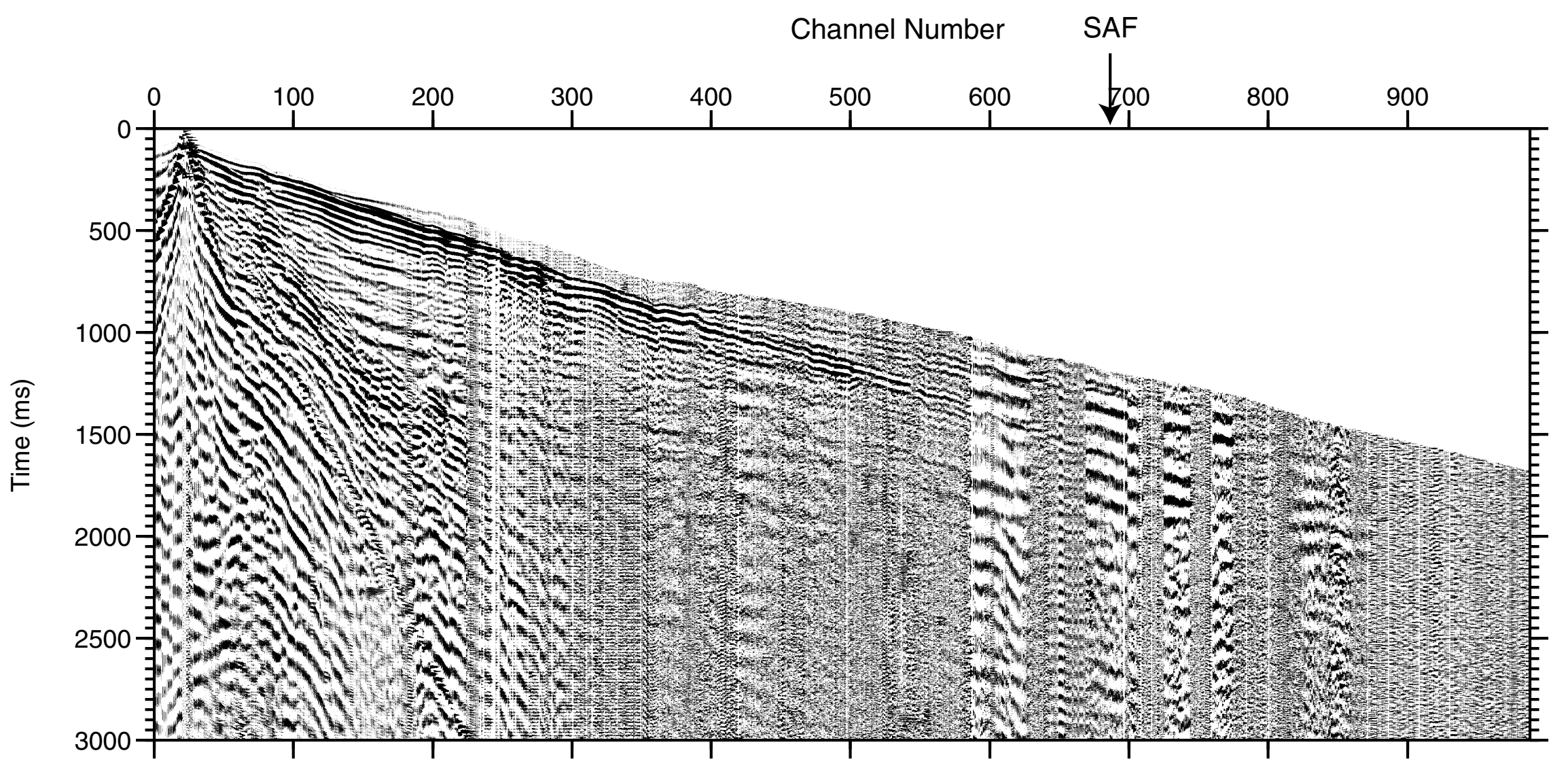

Figure 7. 


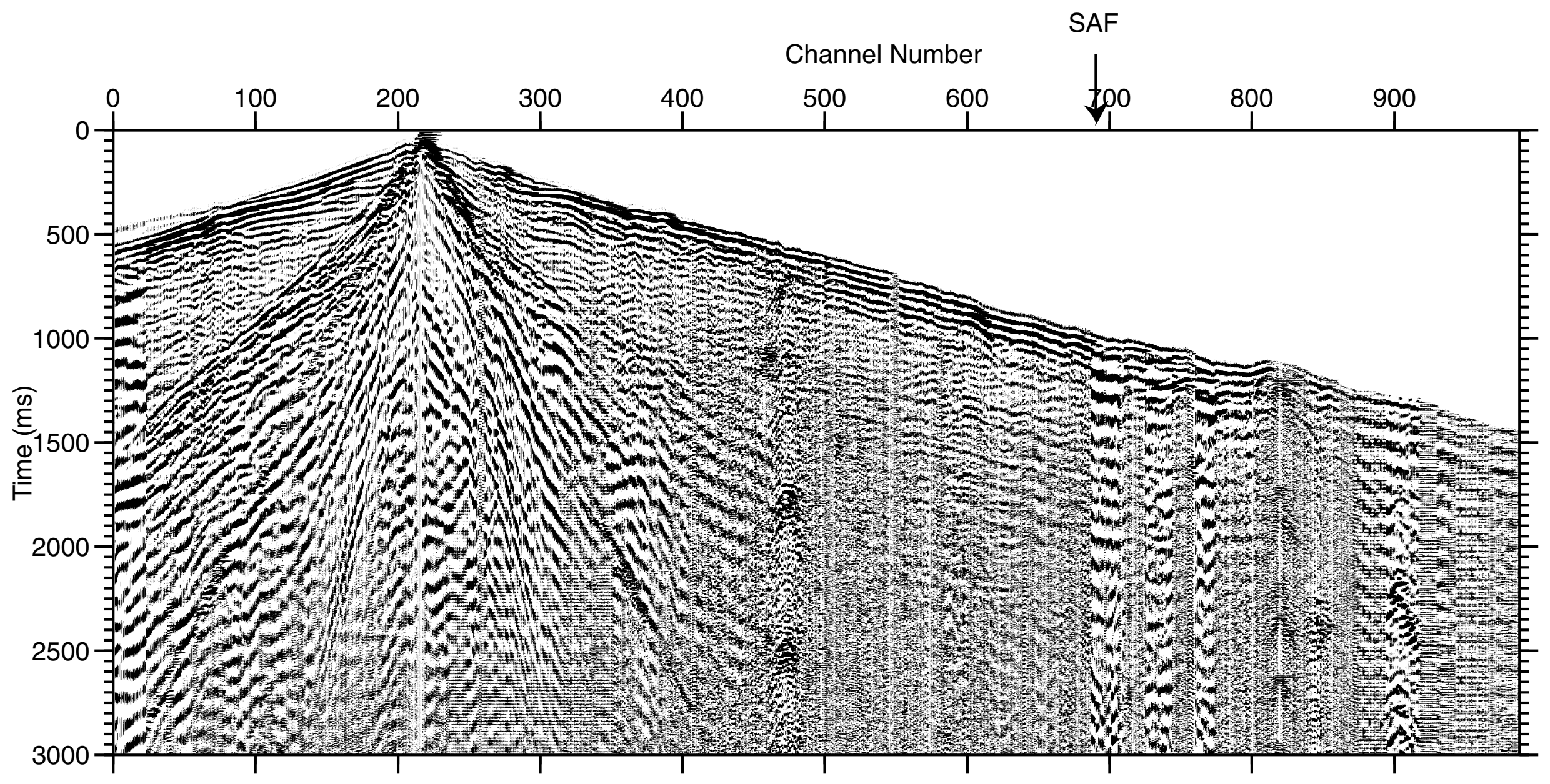

Figure 8. 
Parkfield - Line 1 FFID 1224

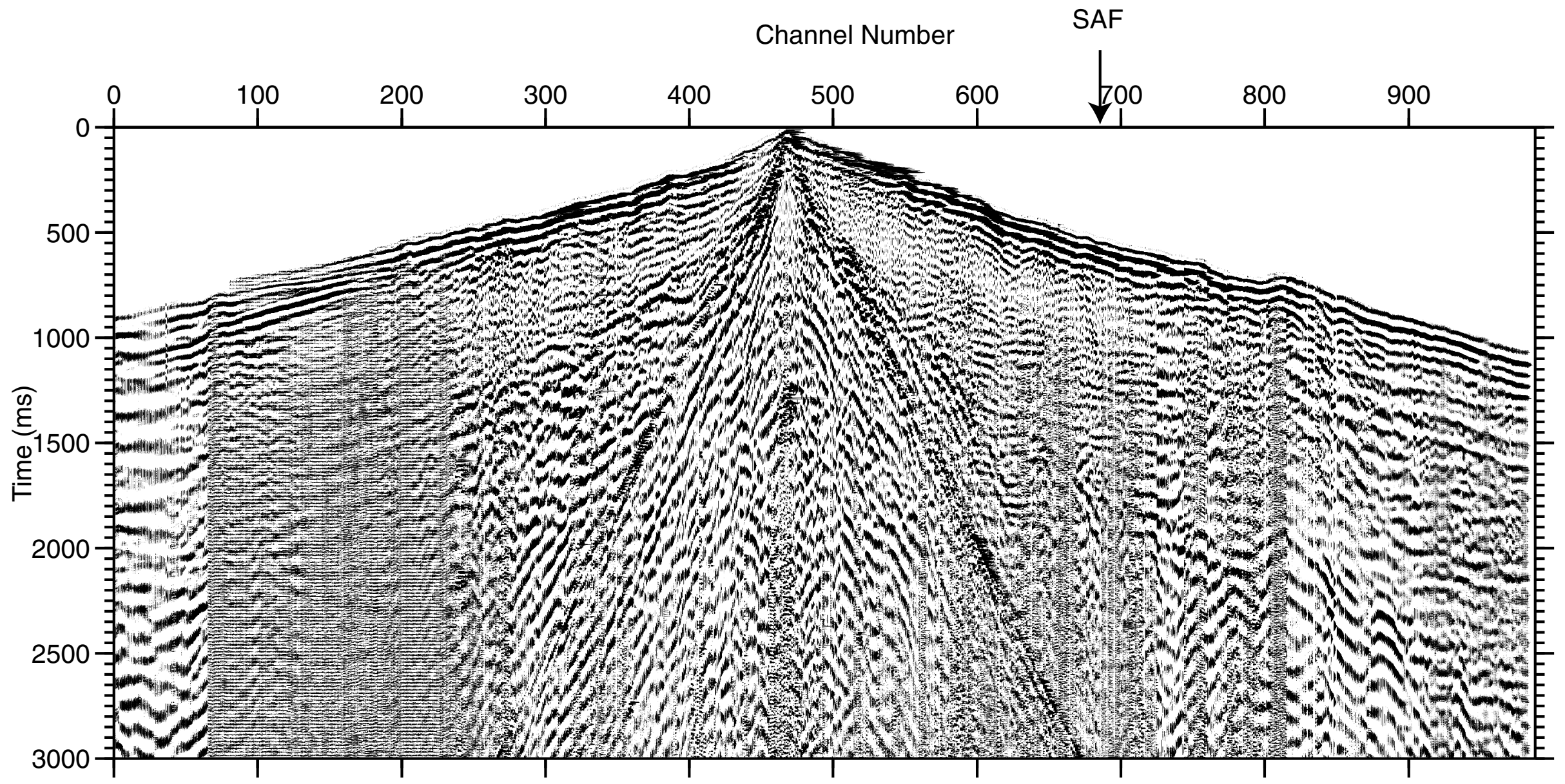

Figure 9. 
Parkfield - Line 1 FFID 1333

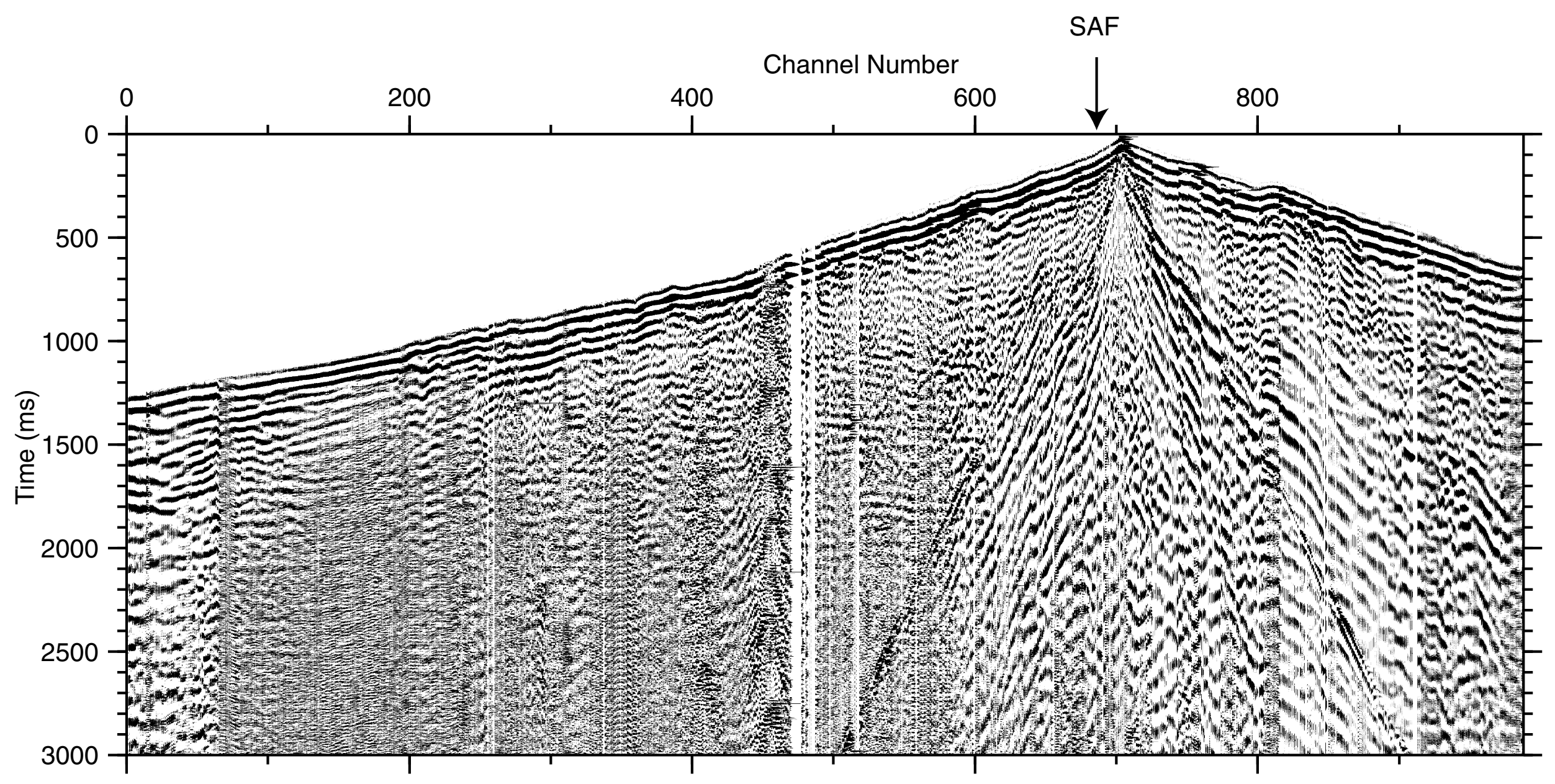

Figure 10. 


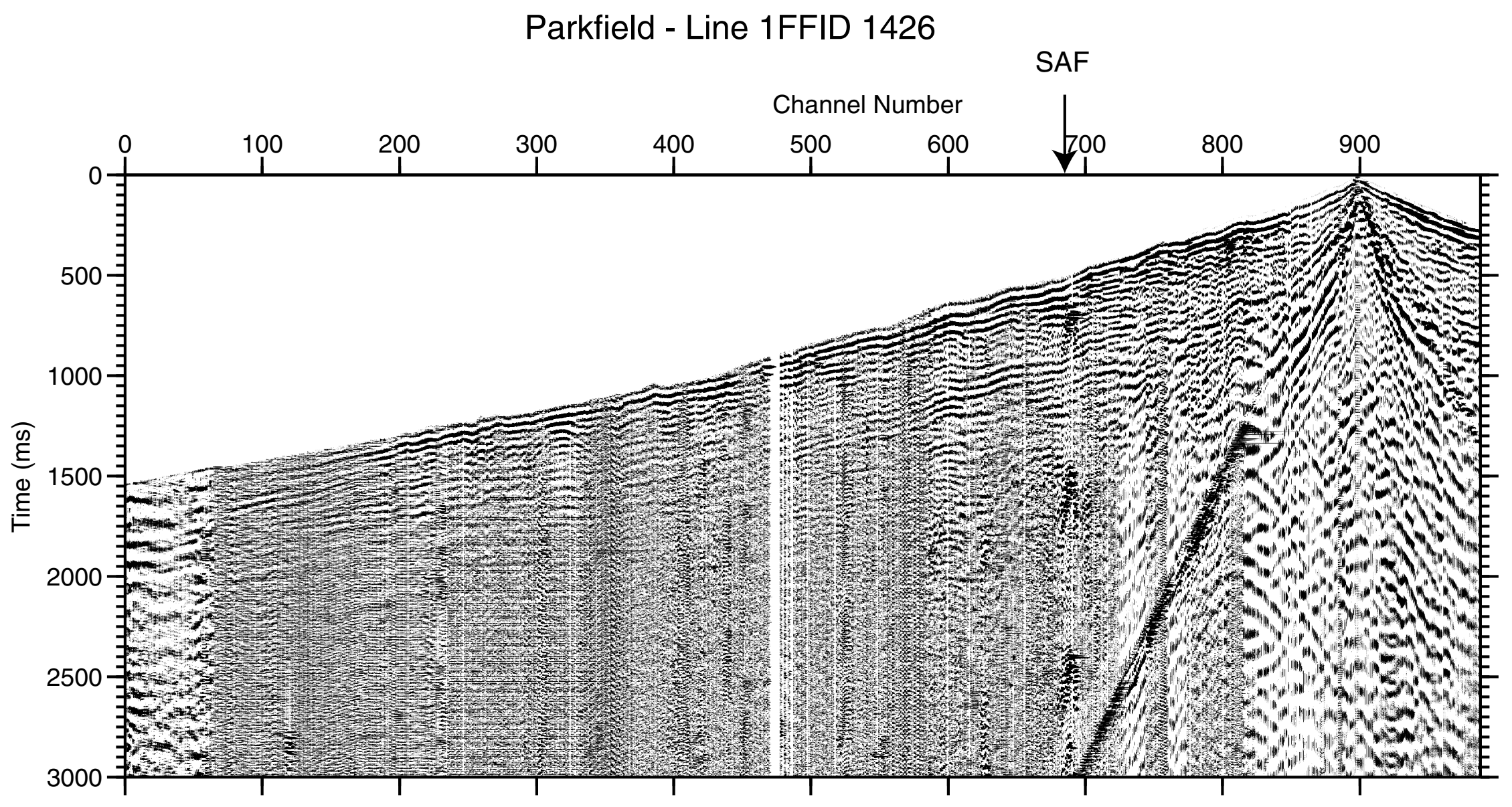

Figure 11. 


\section{Appendix A}

Relative locations and elevations of shots and geophones along main line of Parkfield seismic line.

Distance and lateral variation measurements are relative to first shot, elevation is relative to lowest shot.

\begin{tabular}{|c|c|c|c|c|c|c|}
\hline $\begin{array}{l}\text { Shot/Geophone } \\
\text { number }\end{array}$ & $\begin{array}{c}\text { Geophone } \\
\text { distance }(\mathrm{m})\end{array}$ & $\begin{array}{l}\text { Shot distance } \\
\text { (m) }\end{array}$ & $\begin{array}{c}\text { Geophone } \\
\text { elevation }(\mathrm{m})\end{array}$ & $\begin{array}{l}\text { Shot elevation } \\
\text { (m) }\end{array}$ & $\begin{array}{l}\text { Lateral geophone } \\
\text { variation }(\mathrm{m})\end{array}$ & $\begin{array}{c}\text { Lateral shot } \\
\text { variation }(\mathrm{m})\end{array}$ \\
\hline 1 & 0.14 & 0.00 & 22.27 & 22.44 & 0.72 & 0.00 \\
\hline 2 & 5.43 & & 21.96 & & 0.80 & \\
\hline 3 & 10.73 & 10.72 & 21.65 & 21.57 & 0.88 & -0.11 \\
\hline 4 & 15.73 & & 21.49 & & 0.92 & \\
\hline 5 & 20.73 & 20.41 & 21.33 & 21.30 & 0.96 & -0.08 \\
\hline 6 & 25.76 & & 21.22 & & 1.03 & \\
\hline 7 & 30.79 & & 21.11 & & 1.10 & \\
\hline 8 & 35.75 & & 21.03 & & 1.06 & \\
\hline 9 & 40.70 & 40.37 & 20.95 & 21.07 & 1.02 & 0.12 \\
\hline 10 & 45.72 & & 20.90 & & 1.16 & \\
\hline 11 & 50.73 & 50.64 & 20.85 & 20.91 & 1.31 & 0.05 \\
\hline 12 & 55.69 & & 20.68 & & 1.19 & \\
\hline 13 & 60.66 & 60.79 & 20.52 & 20.62 & 1.08 & 0.16 \\
\hline 14 & 65.67 & & 20.39 & & 0.93 & \\
\hline 15 & 70.68 & 70.71 & 20.25 & 20.37 & 0.77 & -0.18 \\
\hline 16 & 75.67 & & 20.11 & & 0.89 & \\
\hline 17 & 80.65 & 80.39 & 19.96 & 20.14 & 1.01 & 0.09 \\
\hline 18 & 85.62 & & 19.83 & & 1.14 & \\
\hline 19 & 90.58 & 90.96 & 19.70 & 19.78 & 1.27 & 0.36 \\
\hline 20 & 95.64 & & 19.64 & & 1.16 & \\
\hline 21 & 100.69 & 100.24 & 19.58 & 19.74 & 1.06 & 0.00 \\
\hline 22 & 105.53 & & 19.51 & & 1.07 & \\
\hline 23 & 110.37 & 110.74 & 19.44 & 19.58 & 1.08 & 0.27 \\
\hline 24 & 115.51 & & 19.33 & & 1.04 & \\
\hline 25 & 120.64 & 120.52 & 19.21 & 19.36 & 1.00 & 0.00 \\
\hline 26 & 125.67 & & 19.10 & & 1.01 & \\
\hline 27 & 130.69 & 130.43 & 18.99 & 19.08 & 1.03 & 0.00 \\
\hline 28 & 135.69 & & 18.85 & & 0.96 & \\
\hline 29 & 140.70 & 140.31 & 18.71 & 18.89 & 0.90 & -0.22 \\
\hline 30 & 145.71 & & 18.64 & & 0.81 & \\
\hline 31 & 150.72 & 150.10 & 18.56 & 18.67 & 0.73 & -0.10 \\
\hline 32 & 155.75 & & 18.45 & & 0.73 & \\
\hline 33 & 160.79 & 160.49 & 18.33 & 18.40 & 0.72 & -0.17 \\
\hline 34 & 165.76 & & 18.19 & & 0.73 & \\
\hline 35 & 170.74 & & 18.04 & & 0.73 & \\
\hline 36 & 175.77 & & 17.92 & & 0.84 & \\
\hline 37 & 180.80 & 180.49 & 17.79 & 17.95 & 0.95 & -0.07 \\
\hline 38 & 185.81 & & 17.69 & & 0.90 & \\
\hline 39 & 190.82 & 190.55 & 17.58 & 17.76 & 0.85 & -0.22 \\
\hline 40 & 195.78 & & 17.46 & & 1.00 & \\
\hline 41 & 200.74 & 200.65 & 17.34 & 17.47 & 1.14 & -0.04 \\
\hline 42 & 205.76 & & 17.23 & & 1.04 & \\
\hline 43 & 210.77 & 210.41 & 17.13 & 17.26 & 0.94 & -0.15 \\
\hline 44 & 215.83 & & 17.01 & & 1.11 & \\
\hline 45 & 220.89 & 220.45 & 16.89 & 17.06 & 1.28 & 0.41 \\
\hline 46 & 225.93 & & 16.77 & & 1.09 & \\
\hline 47 & 230.98 & 230.54 & 16.65 & 16.80 & 0.91 & 0.10 \\
\hline 48 & 236.44 & & 16.54 & & 1.01 & \\
\hline
\end{tabular}

Appendix A, page 1 


\begin{tabular}{|c|c|c|c|c|c|c|}
\hline 49 & 241.90 & 241.25 & 16.44 & 16.62 & 1.11 & 0.11 \\
\hline 50 & 246.46 & & 16.38 & & 0.95 & \\
\hline 51 & 251.03 & 250.24 & 16.32 & 16.44 & 0.79 & 0.18 \\
\hline 52 & 256.14 & & 16.20 & & 1.00 & \\
\hline 53 & 261.25 & 261.82 & 16.07 & 16.18 & 1.21 & 0.34 \\
\hline 54 & 266.12 & & 15.99 & & 0.86 & \\
\hline 55 & 270.99 & 270.63 & 15.90 & 16.06 & 0.51 & -0.37 \\
\hline 56 & 275.73 & & 15.86 & & 0.91 & \\
\hline 57 & 280.47 & 280.56 & 15.83 & 15.91 & 1.31 & 0.51 \\
\hline 58 & 285.76 & & 15.72 & & 1.28 & \\
\hline 59 & 291.04 & 291.37 & 15.61 & 15.68 & 1.26 & -0.04 \\
\hline 60 & 295.99 & & 15.52 & & 1.13 & \\
\hline 61 & 300.94 & 300.57 & 15.43 & 15.62 & 1.00 & 0.02 \\
\hline 62 & 306.01 & & 15.43 & & 1.12 & \\
\hline 63 & 311.07 & 310.52 & 15.42 & 15.49 & 1.24 & 0.07 \\
\hline 64 & 316.04 & & 15.33 & & 1.20 & \\
\hline 65 & 321.02 & 320.51 & 15.25 & 15.32 & 1.17 & 0.36 \\
\hline 66 & 326.02 & & 15.28 & & 1.13 & \\
\hline 67 & 331.02 & 330.71 & 15.31 & 15.43 & 1.10 & 0.23 \\
\hline 68 & 335.88 & & 15.21 & & 1.43 & \\
\hline 69 & 340.75 & 340.96 & 15.11 & 15.29 & 1.75 & 1.00 \\
\hline 70 & 345.63 & & 15.03 & & 1.75 & \\
\hline 71 & 350.51 & 350.55 & 14.95 & 15.12 & 1.74 & 0.78 \\
\hline 72 & 355.49 & & 14.63 & & 1.61 & \\
\hline 73 & 360.47 & 360.70 & 14.30 & 14.41 & 1.47 & 0.67 \\
\hline 74 & 365.71 & & 13.97 & & 1.08 & \\
\hline 75 & 370.95 & 371.06 & 13.64 & 13.78 & 0.68 & -0.13 \\
\hline 76 & 376.18 & & 13.47 & & 0.71 & \\
\hline 77 & 381.41 & 381.32 & 13.30 & 13.41 & 0.73 & -0.14 \\
\hline 78 & 386.23 & & 13.20 & & 0.75 & \\
\hline 79 & 391.04 & 391.19 & 13.09 & 13.11 & 0.77 & -0.24 \\
\hline 80 & 396.04 & & 12.94 & & 0.66 & \\
\hline 81 & 401.05 & 400.72 & 12.79 & 12.85 & 0.54 & -0.31 \\
\hline 82 & 406.06 & & 12.63 & & 0.62 & \\
\hline 83 & 411.08 & 410.62 & 12.47 & 12.57 & 0.71 & -0.26 \\
\hline 84 & 416.12 & & 12.36 & & 0.64 & \\
\hline 85 & 421.17 & 421.23 & 12.24 & 12.33 & 0.58 & -0.35 \\
\hline 86 & 426.16 & & 12.11 & & 0.65 & \\
\hline 87 & 431.15 & 430.61 & 11.98 & 12.08 & 0.72 & -0.05 \\
\hline 88 & 436.12 & & 11.86 & & 0.61 & \\
\hline 89 & 441.10 & 440.75 & 11.75 & 11.88 & 0.49 & -0.36 \\
\hline 90 & 446.12 & & 11.63 & & 0.48 & \\
\hline 91 & 451.14 & 450.60 & 11.50 & 11.64 & 0.48 & -0.36 \\
\hline 92 & 456.12 & & 11.38 & & 0.53 & \\
\hline 93 & 461.10 & 460.90 & 11.27 & 11.41 & 0.58 & -0.32 \\
\hline 94 & 466.10 & & 11.15 & & 0.63 & \\
\hline 95 & 471.10 & 470.58 & 11.04 & 11.10 & 0.68 & -0.33 \\
\hline 96 & 476.10 & & 10.94 & & 0.66 & \\
\hline 97 & 481.09 & 480.65 & 10.84 & 10.92 & 0.63 & -0.30 \\
\hline 98 & 486.08 & & 10.74 & & 0.68 & \\
\hline 99 & 491.08 & 491.13 & 10.64 & 10.73 & 0.72 & -0.33 \\
\hline 100 & 496.09 & & 10.51 & & 0.75 & \\
\hline 101 & 501.10 & 500.65 & 10.38 & 10.51 & 0.78 & -0.15 \\
\hline 102 & 506.12 & & 10.28 & & 0.85 & \\
\hline
\end{tabular}

Appendix A, page 2 


\begin{tabular}{|c|c|c|c|c|c|c|}
\hline 103 & 511.14 & 510.58 & 10.18 & 10.28 & 0.92 & -0.06 \\
\hline 104 & 516.11 & & 10.06 & & 0.95 & \\
\hline 105 & 521.08 & 520.57 & 9.95 & 10.19 & 0.97 & 0.01 \\
\hline 106 & 526.08 & & 9.84 & & 0.87 & \\
\hline 107 & 531.07 & 530.80 & 9.74 & 9.86 & 0.77 & -0.49 \\
\hline 108 & 536.08 & & 9.62 & & 0.77 & \\
\hline 109 & 541.09 & 540.66 & 9.51 & 9.60 & 0.78 & -0.02 \\
\hline 110 & 546.08 & & 9.41 & & 0.89 & \\
\hline 111 & 551.06 & 550.96 & 9.31 & 9.45 & 1.01 & 0.03 \\
\hline 112 & 556.08 & & 9.18 & & 0.97 & \\
\hline 113 & 561.11 & & 9.06 & & 0.92 & \\
\hline 114 & 566.11 & & 8.95 & & 1.00 & \\
\hline 115 & 571.11 & 570.64 & 8.83 & 9.00 & 1.08 & 0.12 \\
\hline 116 & 576.04 & & 8.71 & & 1.04 & \\
\hline 117 & 580.98 & 580.74 & 8.58 & 8.77 & 1.00 & 0.06 \\
\hline 118 & 586.01 & & 8.46 & & 1.12 & \\
\hline 119 & 591.03 & 590.52 & 8.33 & 8.53 & 1.23 & 0.34 \\
\hline 120 & 596.09 & & 8.20 & & 1.24 & \\
\hline 121 & 601.15 & 600.73 & 8.07 & 8.30 & 1.25 & 0.23 \\
\hline 122 & 606.15 & & 7.98 & & 1.27 & \\
\hline 123 & 611.16 & 610.73 & 7.88 & 8.09 & 1.30 & 0.48 \\
\hline 124 & 616.16 & & 7.80 & & 1.32 & \\
\hline 125 & 621.17 & 620.56 & 7.73 & 7.91 & 1.35 & 0.42 \\
\hline 126 & 626.15 & & 7.60 & & 1.34 & \\
\hline 127 & 631.13 & 630.75 & 7.48 & 7.64 & 1.33 & 0.47 \\
\hline 128 & 636.16 & & 7.40 & & 1.29 & \\
\hline 129 & 641.19 & 640.53 & 7.32 & 7.48 & 1.25 & 0.36 \\
\hline 130 & 646.15 & & 7.23 & & 1.31 & \\
\hline 131 & 651.11 & 650.63 & 7.14 & 7.29 & 1.36 & 0.40 \\
\hline 132 & 656.12 & & 7.02 & & 1.31 & \\
\hline 133 & 661.13 & 660.38 & 6.90 & 7.06 & 1.25 & 0.43 \\
\hline 134 & 666.12 & & 6.80 & & 1.32 & \\
\hline 135 & 671.12 & 670.62 & 6.71 & 6.88 & 1.40 & 0.30 \\
\hline 136 & 676.15 & & 6.60 & & 1.34 & \\
\hline 137 & 681.18 & 680.81 & 6.49 & 6.70 & 1.29 & 0.40 \\
\hline 138 & 686.15 & & 6.40 & & 1.32 & \\
\hline 139 & 691.12 & 690.70 & 6.30 & 6.43 & 1.35 & 0.47 \\
\hline 140 & 696.14 & & 6.22 & & 1.38 & \\
\hline 141 & 701.17 & 700.46 & 6.13 & 6.31 & 1.40 & 0.61 \\
\hline 142 & 706.18 & & 6.04 & & 1.44 & \\
\hline 143 & 711.19 & 710.75 & 5.95 & 6.11 & 1.49 & 0.71 \\
\hline 144 & 716.17 & & 5.85 & & 1.43 & \\
\hline 145 & 721.16 & 720.65 & 5.75 & 5.90 & 1.37 & 0.46 \\
\hline 146 & 726.16 & & 5.65 & & 1.38 & \\
\hline 147 & 731.17 & 730.50 & 5.55 & 5.69 & 1.38 & 0.54 \\
\hline 148 & 736.19 & & 5.44 & & 1.39 & \\
\hline 149 & 741.21 & 740.70 & 5.32 & 5.50 & 1.41 & 0.41 \\
\hline 150 & 746.18 & & 5.24 & & 1.43 & \\
\hline 151 & 751.16 & 750.72 & 5.17 & 5.36 & 1.44 & 0.69 \\
\hline 152 & 756.18 & & 5.06 & & 1.45 & \\
\hline 153 & 761.20 & 760.72 & 4.96 & 5.13 & 1.45 & 0.56 \\
\hline 154 & 766.21 & & 4.84 & & 1.49 & \\
\hline 155 & 771.21 & & 4.73 & & 1.52 & \\
\hline 156 & 776.21 & & 4.63 & & 1.56 & \\
\hline
\end{tabular}

Appendix A, page 3 


\begin{tabular}{|c|c|c|c|c|c|c|}
\hline 157 & 781.22 & 780.51 & 4.54 & 4.68 & 1.59 & 0.62 \\
\hline 158 & 786.20 & & 4.46 & & 1.51 & \\
\hline 159 & 791.19 & 790.58 & 4.37 & 4.58 & 1.43 & 0.55 \\
\hline 160 & 796.20 & & 4.32 & & 1.56 & \\
\hline 161 & 801.21 & 800.85 & 4.26 & 4.46 & 1.69 & 0.81 \\
\hline 162 & 806.21 & & 4.17 & & 1.56 & \\
\hline 163 & 811.20 & 810.39 & 4.07 & 4.27 & 1.42 & 0.49 \\
\hline 164 & 816.23 & & 4.01 & & 1.40 & \\
\hline 165 & 821.25 & 820.59 & 3.96 & 4.15 & 1.38 & 0.53 \\
\hline 166 & 826.25 & & 3.90 & & 1.39 & \\
\hline 167 & 831.25 & 830.65 & 3.84 & 4.04 & 1.41 & 0.63 \\
\hline 168 & 836.25 & & 3.80 & & 1.38 & \\
\hline 169 & 841.25 & 840.64 & 3.76 & 3.97 & 1.34 & 0.51 \\
\hline 170 & 846.26 & & 3.70 & & 1.37 & \\
\hline 171 & 851.27 & 850.83 & 3.63 & 3.82 & 1.39 & 0.50 \\
\hline 172 & 856.24 & & 3.56 & & 1.39 & \\
\hline 173 & 861.22 & 860.63 & 3.50 & 3.69 & 1.40 & 0.67 \\
\hline 174 & 866.28 & & 3.46 & & 1.38 & \\
\hline 175 & 871.34 & 870.78 & 3.41 & 3.68 & 1.35 & 0.42 \\
\hline 176 & 876.27 & & 3.35 & & 1.38 & \\
\hline 177 & 881.19 & 880.79 & 3.29 & 3.43 & 1.40 & 0.67 \\
\hline 178 & 886.11 & & 3.24 & & 1.47 & \\
\hline 179 & 891.04 & 891.08 & 3.20 & 3.44 & 1.54 & 0.70 \\
\hline 180 & 896.19 & & 3.29 & & 1.55 & \\
\hline 181 & 901.35 & 900.80 & 3.38 & 3.57 & 1.56 & 0.88 \\
\hline 182 & 905.57 & & 3.36 & & 1.79 & \\
\hline 183 & 909.79 & 909.68 & 3.34 & 3.52 & 2.02 & 1.01 \\
\hline 184 & 915.58 & & 3.39 & & 1.84 & \\
\hline 185 & 921.38 & 921.27 & 3.44 & 3.57 & 1.66 & 0.94 \\
\hline 186 & 926.45 & & 3.41 & & 1.68 & \\
\hline 187 & 931.52 & & 3.38 & & 1.70 & \\
\hline 188 & 936.31 & & 3.35 & & 1.87 & \\
\hline 189 & 941.09 & 940.62 & 3.33 & 3.54 & 2.04 & 0.84 \\
\hline 190 & 945.97 & & 3.34 & & 1.73 & \\
\hline 191 & 950.84 & 950.28 & 3.35 & 3.54 & 1.43 & 0.47 \\
\hline 192 & 955.98 & & 3.24 & & 1.23 & \\
\hline 193 & 961.12 & 960.17 & 3.14 & 3.39 & 1.04 & 0.26 \\
\hline 194 & 965.86 & & 3.20 & & 1.12 & \\
\hline 195 & 970.59 & 970.66 & 3.26 & 3.42 & 1.20 & 0.39 \\
\hline 196 & 975.77 & & 2.57 & & 1.22 & \\
\hline 197 & 980.94 & 980.58 & 1.87 & 2.01 & 1.24 & 0.42 \\
\hline 198 & 986.05 & & 0.94 & & 1.29 & \\
\hline 199 & 991.17 & 990.82 & 0.00 & 0.24 & 1.33 & 0.23 \\
\hline 200 & 996.22 & & 0.63 & & 1.22 & \\
\hline 201 & 1001.27 & 1000.70 & 1.25 & 0.94 & 1.10 & 0.27 \\
\hline 202 & 1006.12 & & 0.73 & & 1.28 & \\
\hline 203 & 1010.96 & 1010.60 & 0.20 & 0.35 & 1.46 & 0.45 \\
\hline 204 & 1017.24 & & 0.28 & & 1.75 & \\
\hline 205 & 1023.51 & 1022.13 & 0.36 & 0.01 & 2.05 & 1.03 \\
\hline 206 & 1027.17 & & 1.49 & & 1.61 & \\
\hline 207 & 1030.83 & 1030.59 & 2.63 & 2.85 & 1.17 & 0.28 \\
\hline 208 & 1035.85 & & 2.64 & & 1.13 & \\
\hline 209 & 1040.87 & 1040.66 & 2.65 & 2.83 & 1.09 & 0.26 \\
\hline 210 & 1045.85 & & 2.56 & & 1.01 & \\
\hline
\end{tabular}

Appendix A, page 4 


\begin{tabular}{|c|c|c|c|c|c|c|}
\hline 211 & 1050.83 & 1050.68 & 2.48 & 2.68 & 0.93 & 0.00 \\
\hline 212 & 1055.82 & & 2.50 & & 0.81 & \\
\hline 213 & 1060.80 & & 2.53 & & 0.70 & \\
\hline 214 & 1065.82 & & 2.37 & & 0.76 & \\
\hline 215 & 1070.83 & 1070.52 & 2.21 & 2.40 & 0.82 & 0.00 \\
\hline 216 & 1075.60 & & 1.90 & & 0.67 & \\
\hline 217 & 1080.37 & 1080.53 & 1.60 & 1.81 & 0.53 & -0.49 \\
\hline 218 & 1085.53 & & 2.05 & & 0.71 & \\
\hline 219 & 1090.70 & 1090.76 & 2.51 & 2.62 & 0.90 & 0.11 \\
\hline 220 & 1095.72 & & 3.30 & & 1.00 & \\
\hline 221 & 1100.74 & 1100.59 & 4.10 & 4.21 & 1.10 & 0.14 \\
\hline 222 & 1105.59 & & 5.61 & & 1.03 & \\
\hline 223 & 1110.44 & 1110.60 & 7.13 & 7.27 & 0.96 & 0.04 \\
\hline 224 & 1115.50 & & 8.92 & & 0.83 & \\
\hline 225 & 1120.57 & 1119.64 & 10.70 & 10.52 & 0.71 & -0.07 \\
\hline 226 & 1125.44 & & 11.30 & & 0.72 & \\
\hline 227 & 1130.32 & 1129.74 & 11.91 & 12.07 & 0.74 & -0.09 \\
\hline 228 & 1135.37 & & 11.25 & & -0.09 & \\
\hline 229 & 1140.42 & 1139.76 & 10.60 & 10.81 & -0.92 & -1.77 \\
\hline 230 & 1145.15 & & 9.67 & & -1.10 & \\
\hline 231 & 1149.87 & 1149.70 & 8.75 & 8.86 & -1.28 & -2.31 \\
\hline 232 & 1154.73 & & 7.64 & & -1.27 & \\
\hline 233 & 1159.59 & 1159.28 & 6.52 & 6.66 & -1.26 & -2.33 \\
\hline 234 & 1164.41 & & 5.39 & & -1.39 & \\
\hline 235 & 1169.45 & 1169.37 & 4.87 & 5.07 & -1.43 & -2.37 \\
\hline 236 & 1174.63 & & 4.66 & & -1.61 & \\
\hline 237 & 1179.52 & 1179.30 & 4.59 & 4.85 & -1.69 & -2.77 \\
\hline 238 & 1184.52 & & 4.92 & & -1.66 & \\
\hline 239 & 1189.51 & 1189.13 & 5.15 & 5.44 & -1.61 & -2.40 \\
\hline 240 & 1194.69 & & 4.97 & & -1.78 & \\
\hline 241 & 1199.61 & 1199.49 & 4.50 & 4.84 & -1.55 & -2.35 \\
\hline 242 & 1204.52 & & 4.13 & & -1.76 & \\
\hline 243 & 1209.44 & 1209.27 & 3.87 & 4.16 & -1.77 & -2.78 \\
\hline 244 & 1214.57 & & 3.73 & & -1.70 & \\
\hline 245 & 1219.62 & 1219.47 & 3.53 & 3.78 & -1.64 & -2.60 \\
\hline 246 & 1224.68 & & 4.14 & & -1.82 & \\
\hline 247 & 1229.60 & 1229.15 & 4.81 & 4.97 & -1.68 & -2.66 \\
\hline 248 & 1234.41 & & 5.87 & & -1.80 & \\
\hline 249 & 1239.45 & 1238.59 & 7.18 & 7.14 & -1.74 & -2.49 \\
\hline 250 & 1244.28 & & 8.36 & & -1.85 & \\
\hline 251 & 1249.19 & 1248.75 & 9.33 & 9.42 & -1.66 & -2.58 \\
\hline 252 & 1254.15 & & 10.05 & & -1.71 & \\
\hline 253 & 1259.21 & 1259.04 & 10.41 & 10.63 & -1.67 & -2.55 \\
\hline 254 & 1264.18 & & 10.12 & & -1.53 & \\
\hline 255 & 1268.78 & 1269.08 & 9.46 & 9.67 & -1.13 & -2.01 \\
\hline 256 & 1274.06 & & 8.51 & & -0.91 & \\
\hline 257 & 1279.16 & 1279.09 & 7.75 & 7.97 & -0.94 & -1.97 \\
\hline 258 & 1283.94 & & 7.25 & & -1.23 & \\
\hline 259 & 1288.96 & 1289.03 & 6.98 & 7.16 & -1.60 & -2.58 \\
\hline 260 & 1294.04 & & 7.15 & & -1.54 & \\
\hline 261 & 1299.05 & 1298.61 & 7.73 & 7.94 & -1.26 & -2.25 \\
\hline 262 & 1303.91 & & 8.46 & & -1.10 & \\
\hline 263 & 1308.89 & & 9.45 & & -0.63 & \\
\hline 264 & 1313.64 & & 10.56 & & -0.78 & \\
\hline
\end{tabular}

Appendix A, page 5 


\begin{tabular}{|c|c|c|c|c|c|c|}
\hline 265 & 1318.73 & 1318.94 & 11.46 & 11.66 & -0.84 & -1.65 \\
\hline 266 & 1323.70 & & 11.66 & & -1.04 & \\
\hline 267 & 1328.72 & 1329.15 & 11.32 & 11.48 & -1.23 & -2.28 \\
\hline 268 & 1333.60 & & 10.63 & & -1.62 & \\
\hline 269 & 1338.79 & 1338.24 & 9.00 & 9.44 & -2.75 & -3.65 \\
\hline 270 & 1342.91 & & 5.92 & & -2.05 & \\
\hline 271 & 1348.29 & 1348.35 & 7.76 & 8.26 & -2.15 & -3.05 \\
\hline 272 & 1353.37 & & 8.13 & & -2.10 & \\
\hline 273 & 1358.05 & 1358.51 & 7.59 & 8.08 & -2.41 & -3.26 \\
\hline 274 & 1363.33 & & 8.40 & & -2.55 & \\
\hline 275 & 1368.02 & 1368.81 & 8.84 & 9.20 & -2.60 & -3.56 \\
\hline 276 & 1373.37 & & 9.24 & & -2.83 & \\
\hline 277 & 1377.87 & 1378.79 & 9.79 & 10.04 & -3.08 & -3.87 \\
\hline 278 & 1383.50 & & 10.59 & & -3.04 & \\
\hline 279 & 1388.51 & 1389.15 & 10.78 & 10.99 & -3.21 & -3.90 \\
\hline 280 & 1393.45 & & 10.99 & & -3.20 & \\
\hline 281 & 1398.54 & & 11.13 & & -3.17 & \\
\hline 282 & 1403.56 & & 11.41 & & -3.26 & \\
\hline 283 & 1408.52 & 1409.32 & 11.43 & 11.70 & -3.95 & -4.69 \\
\hline 284 & 1413.60 & & 11.52 & & -3.54 & \\
\hline 285 & 1418.23 & 1419.22 & 11.75 & 12.00 & -3.60 & -4.47 \\
\hline 286 & 1423.57 & & 11.92 & & -3.74 & \\
\hline 287 & 1428.42 & 1428.88 & 12.10 & 12.33 & -3.85 & -4.80 \\
\hline 288 & 1433.45 & & 12.27 & & -3.93 & \\
\hline 289 & 1438.50 & 1438.93 & 12.44 & 12.68 & -4.03 & -4.98 \\
\hline 290 & 1443.46 & & 12.64 & & -4.30 & \\
\hline 291 & 1448.47 & 1448.67 & 12.80 & 13.07 & -4.25 & -5.24 \\
\hline 292 & 1453.50 & & 12.96 & & -4.62 & \\
\hline 293 & 1458.34 & 1458.58 & 13.11 & 13.33 & -4.72 & -5.64 \\
\hline 294 & 1463.41 & & 13.31 & & -4.79 & \\
\hline 295 & 1468.42 & 1468.53 & 13.49 & 13.72 & -4.90 & -5.79 \\
\hline 296 & 1473.34 & & 13.59 & & -5.08 & \\
\hline 297 & 1478.34 & 1478.77 & 13.72 & 13.97 & -4.92 & -5.81 \\
\hline 298 & 1483.41 & & 13.88 & & -5.04 & \\
\hline 299 & 1488.39 & 1488.62 & 14.08 & 14.31 & -5.20 & -6.09 \\
\hline 300 & 1493.30 & & 14.28 & & -5.19 & \\
\hline 301 & 1498.43 & 1498.77 & 14.54 & 14.78 & -5.52 & -6.00 \\
\hline 302 & 1503.40 & & 14.85 & & -5.60 & \\
\hline 303 & 1508.37 & 1508.80 & 15.00 & 15.23 & -5.63 & -6.25 \\
\hline 304 & 1513.49 & & 15.35 & & -5.77 & \\
\hline 305 & 1518.48 & 1518.80 & 15.42 & 15.64 & -5.71 & -6.46 \\
\hline 306 & 1523.51 & & 15.75 & & -5.64 & \\
\hline 307 & 1528.52 & & 15.91 & & -5.75 & \\
\hline 308 & 1533.37 & & 16.10 & & -5.79 & \\
\hline 309 & 1538.26 & 1538.85 & 16.31 & 16.45 & -5.94 & -6.55 \\
\hline 310 & 1543.69 & & 16.44 & & -5.57 & \\
\hline 311 & 1548.27 & 1548.96 & 16.63 & 16.78 & -6.17 & -6.84 \\
\hline 312 & 1553.34 & & 16.78 & & -6.16 & \\
\hline 313 & 1558.59 & 1558.93 & 17.19 & 17.30 & -6.25 & -6.91 \\
\hline 314 & 1563.44 & & 17.51 & & -6.24 & \\
\hline 315 & 1568.71 & 1568.74 & 17.85 & 17.98 & -6.57 & -7.13 \\
\hline 316 & 1573.48 & & 18.09 & & -6.34 & \\
\hline 317 & 1578.56 & 1579.11 & 18.39 & 18.60 & -6.11 & -6.94 \\
\hline 318 & 1583.15 & & 18.54 & & -6.51 & \\
\hline
\end{tabular}




\begin{tabular}{|c|c|c|c|c|c|c|}
\hline 319 & 1588.52 & 1588.94 & 19.06 & 19.31 & -6.72 & -7.08 \\
\hline 320 & 1593.49 & & 19.46 & & -6.73 & \\
\hline 321 & 1598.61 & 1598.97 & 19.65 & 19.84 & -6.71 & -7.37 \\
\hline 322 & 1603.64 & & 19.70 & & -6.84 & \\
\hline 323 & 1608.44 & 1608.65 & 19.92 & 20.14 & -6.84 & -7.38 \\
\hline 324 & 1613.51 & & 20.13 & & -6.71 & \\
\hline 325 & 1618.63 & 1619.14 & 20.34 & 20.57 & -7.10 & -7.51 \\
\hline 326 & 1623.70 & & 20.46 & & -6.69 & \\
\hline 327 & 1628.49 & & 20.79 & & -7.03 & \\
\hline 328 & 1633.46 & & 20.97 & & -7.09 & \\
\hline 329 & 1638.45 & 1639.02 & 21.16 & 21.41 & -7.03 & -7.62 \\
\hline 330 & 1643.52 & & 21.20 & & -7.30 & \\
\hline 331 & 1648.34 & 1648.92 & 21.39 & 21.64 & -7.28 & -7.54 \\
\hline 332 & 1653.72 & & 21.53 & & -7.32 & \\
\hline 333 & 1658.97 & 1659.65 & 21.66 & 21.93 & -7.11 & -7.47 \\
\hline 334 & 1663.36 & & 21.79 & & -6.77 & \\
\hline 335 & 1668.15 & 1669.17 & 21.89 & 22.19 & -6.92 & -7.49 \\
\hline 336 & 1673.07 & & 22.08 & & -7.64 & \\
\hline 337 & 1678.11 & 1679.32 & 22.23 & 22.59 & -7.29 & -8.01 \\
\hline 338 & 1683.78 & & 22.41 & & -7.43 & \\
\hline 339 & 1688.61 & 1689.31 & 22.56 & 22.83 & -7.39 & -7.88 \\
\hline 340 & 1693.27 & & 22.78 & & -7.28 & \\
\hline 341 & 1698.21 & 1699.37 & 22.96 & 23.24 & -7.37 & -7.85 \\
\hline 342 & 1702.91 & & 23.26 & & -6.96 & \\
\hline 343 & 1708.32 & 1709.41 & 23.66 & 23.82 & -7.29 & -8.11 \\
\hline 344 & 1712.89 & & 24.06 & & -6.82 & \\
\hline 345 & 1718.23 & 1719.27 & 24.81 & 25.05 & -6.98 & -7.34 \\
\hline 346 & 1723.38 & & 25.77 & & -6.98 & \\
\hline 347 & 1727.96 & 1728.95 & 26.91 & 27.19 & -6.29 & -6.90 \\
\hline 348 & 1732.89 & & 28.10 & & -5.92 & \\
\hline 349 & 1737.71 & 1738.45 & 29.20 & 29.30 & -5.65 & -6.22 \\
\hline 350 & 1742.81 & & 30.23 & & -5.36 & \\
\hline 351 & 1747.41 & 1748.13 & 31.06 & 31.17 & -5.44 & -6.14 \\
\hline 352 & 1752.47 & & 31.82 & & -5.47 & \\
\hline 353 & 1757.52 & 1758.10 & 32.62 & 32.73 & -5.33 & -6.10 \\
\hline 354 & 1763.16 & & 33.45 & & -5.49 & \\
\hline 355 & 1767.37 & 1767.82 & 34.17 & 34.27 & -5.90 & -6.77 \\
\hline 356 & 1772.27 & & 35.20 & & -4.87 & \\
\hline 357 & 1776.95 & 1776.60 & 36.23 & 36.25 & -3.12 & -3.99 \\
\hline 358 & 1781.70 & & 36.94 & & -2.00 & \\
\hline 359 & 1786.20 & 1786.78 & 37.05 & 37.23 & -1.91 & -2.63 \\
\hline 360 & 1791.55 & & 36.75 & & -0.93 & \\
\hline 361 & 1796.24 & 1796.11 & 35.94 & 36.32 & -0.66 & -1.73 \\
\hline 362 & 1801.15 & & 34.68 & & -0.64 & \\
\hline 363 & 1806.07 & 1806.16 & 33.43 & 33.91 & -0.62 & -1.66 \\
\hline 364 & 1810.94 & & 32.42 & & -1.09 & \\
\hline 365 & 1815.68 & 1815.68 & 31.01 & 31.48 & -0.20 & -0.99 \\
\hline 366 & 1821.15 & & 30.05 & & -1.11 & \\
\hline 367 & 1825.97 & 1825.46 & 29.38 & 29.80 & -1.20 & -1.66 \\
\hline 368 & 1831.09 & & 29.02 & & -1.27 & \\
\hline 369 & 1836.23 & 1835.51 & 28.95 & 29.40 & -1.43 & -1.93 \\
\hline 370 & 1841.11 & & 28.68 & & -1.54 & \\
\hline 371 & 1846.18 & 1845.58 & 28.15 & 28.61 & -1.84 & -2.31 \\
\hline 372 & 1851.37 & & 27.51 & & -1.62 & \\
\hline
\end{tabular}

Appendix A, page 7 


\begin{tabular}{|c|c|c|c|c|c|c|}
\hline 373 & 1856.29 & 1855.88 & 27.44 & 27.83 & -1.53 & -2.25 \\
\hline 374 & 1859.58 & & 27.67 & & -1.71 & \\
\hline 375 & 1866.28 & 1866.69 & 28.22 & 28.76 & -2.02 & -2.96 \\
\hline 376 & 1870.91 & & 28.37 & & -2.28 & \\
\hline 377 & 1875.86 & 1875.88 & 27.56 & 28.13 & -1.46 & -2.26 \\
\hline 378 & 1881.06 & & 26.92 & & -1.89 & \\
\hline 379 & 1885.91 & 1885.05 & 25.90 & 26.43 & -2.06 & -2.56 \\
\hline 380 & 1891.05 & & 24.87 & & -2.23 & \\
\hline 381 & 1895.81 & 1895.15 & 23.89 & 24.46 & -2.56 & -3.04 \\
\hline 382 & 1900.52 & & 22.59 & & -2.47 & \\
\hline 383 & 1904.99 & 1904.07 & 20.99 & 21.71 & -2.81 & -3.44 \\
\hline 384 & 1910.26 & & 19.68 & & -2.72 & \\
\hline 385 & 1915.12 & 1914.84 & 18.66 & 19.01 & -2.95 & -3.89 \\
\hline 386 & 1920.09 & & 18.07 & & -3.26 & \\
\hline 387 & 1924.88 & 1923.91 & 17.78 & 18.13 & -3.63 & -4.26 \\
\hline 388 & 1930.10 & & 17.84 & & -3.79 & \\
\hline 389 & 1934.76 & 1933.81 & 17.87 & 18.03 & -3.93 & -4.50 \\
\hline 390 & 1940.23 & & 18.09 & & -3.73 & \\
\hline 391 & 1945.27 & 1945.23 & 18.17 & 18.46 & -3.98 & -4.40 \\
\hline 392 & 1950.13 & & 18.25 & & -3.96 & \\
\hline 393 & 1955.10 & 1954.65 & 18.45 & 18.67 & -3.83 & -4.72 \\
\hline 394 & 1960.16 & & 18.68 & & -3.85 & \\
\hline 395 & 1964.76 & 1963.77 & 18.95 & 19.11 & -4.08 & -4.70 \\
\hline 396 & 1970.12 & & 19.13 & & -3.86 & \\
\hline 397 & 1974.98 & 1973.35 & 19.32 & 19.57 & -3.77 & -4.60 \\
\hline 398 & 1980.17 & & 19.38 & & -3.84 & \\
\hline 399 & 1984.81 & 1983.87 & 19.46 & 19.74 & -4.07 & -4.92 \\
\hline 400 & 1989.97 & & 19.47 & & -3.93 & \\
\hline 401 & 1994.95 & 1993.57 & 19.51 & 19.74 & -3.82 & -4.71 \\
\hline 402 & 1999.90 & & 19.46 & & -3.99 & \\
\hline 403 & 2004.88 & 2003.27 & 19.64 & 19.79 & -4.22 & -5.14 \\
\hline 404 & 2009.93 & & 19.64 & & -4.42 & \\
\hline 405 & 2014.96 & 2013.90 & 19.60 & 19.87 & -4.82 & -5.64 \\
\hline 406 & 2019.78 & & 19.46 & & -4.44 & \\
\hline 407 & 2024.90 & 2023.69 & 19.57 & 19.79 & -4.75 & -6.02 \\
\hline 408 & 2029.99 & & 19.49 & & -4.61 & \\
\hline 409 & 2034.15 & 2032.90 & 19.33 & 19.57 & -4.93 & -5.73 \\
\hline 410 & 2039.80 & & 19.42 & & -4.53 & \\
\hline 411 & 2044.99 & 2043.12 & 19.11 & 19.35 & -4.41 & -5.03 \\
\hline 412 & 2050.04 & & 18.77 & & -4.36 & \\
\hline 413 & 2054.96 & 2054.09 & 18.26 & 18.55 & -4.20 & -4.87 \\
\hline 414 & 2059.89 & & 18.06 & & -4.47 & \\
\hline 415 & 2064.59 & 2064.33 & 17.46 & 17.58 & -4.92 & -5.51 \\
\hline 416 & 2069.83 & & 16.47 & & -4.72 & \\
\hline 417 & 2074.62 & 2074.92 & 15.52 & 15.85 & -4.57 & -5.47 \\
\hline 418 & 2080.09 & & 15.53 & & -4.75 & \\
\hline 419 & 2085.07 & 2085.03 & 16.09 & 16.42 & -4.71 & -5.65 \\
\hline 420 & 2090.10 & & 16.42 & & -4.72 & \\
\hline 421 & 2095.02 & 2094.43 & 16.64 & 16.97 & -5.04 & -6.01 \\
\hline 422 & 2099.80 & & 17.27 & & -5.44 & \\
\hline 423 & 2104.79 & 2104.85 & 17.52 & 17.81 & -5.35 & -6.28 \\
\hline 424 & 2109.68 & & 17.62 & & -5.62 & \\
\hline 425 & 2114.73 & 2114.29 & 17.74 & 17.98 & -5.89 & -7.18 \\
\hline 426 & 2119.59 & & 17.84 & & -6.31 & \\
\hline
\end{tabular}

Appendix A, page 8 


\begin{tabular}{|c|c|c|c|c|c|c|}
\hline 427 & 2124.79 & 2124.50 & 17.96 & 18.20 & -6.21 & -6.97 \\
\hline 428 & 2130.15 & & 17.91 & & -6.07 & \\
\hline 429 & 2134.73 & 2134.37 & 17.87 & 18.12 & -6.62 & -7.59 \\
\hline 430 & 2139.61 & & 17.49 & & -6.59 & \\
\hline 431 & 2144.27 & 2144.23 & 17.32 & 17.67 & -6.67 & -7.89 \\
\hline 432 & 2149.49 & & 17.23 & & -6.54 & \\
\hline 433 & 2154.49 & 2154.09 & 17.16 & 17.44 & -6.45 & -7.32 \\
\hline 434 & 2159.43 & & 16.93 & & -6.81 & \\
\hline 435 & 2164.48 & 2164.96 & 16.34 & 16.46 & -7.29 & -8.47 \\
\hline 436 & 2169.92 & & 15.06 & & -6.90 & \\
\hline 437 & 2174.32 & 2174.70 & 13.82 & 14.02 & -7.65 & -8.33 \\
\hline 438 & 2180.77 & & 15.11 & & -7.67 & \\
\hline 439 & 2184.30 & 2184.36 & 16.19 & 16.36 & -7.47 & -8.44 \\
\hline 440 & 2189.35 & & 17.40 & & -7.30 & \\
\hline 441 & 2194.25 & 2194.03 & 17.97 & 18.11 & -6.92 & -8.55 \\
\hline 442 & 2199.50 & & 18.18 & & -7.13 & \\
\hline 443 & 2204.51 & 2203.87 & 17.91 & 18.15 & -7.65 & -8.99 \\
\hline 444 & 2209.58 & & 17.46 & & -8.33 & \\
\hline 445 & 2214.43 & 2214.95 & 17.55 & 17.85 & -7.98 & -9.25 \\
\hline 446 & 2219.29 & & 17.30 & & -7.08 & \\
\hline 447 & 2224.49 & 2223.89 & 17.59 & 17.97 & -6.85 & -8.54 \\
\hline 448 & 2229.45 & & 17.91 & & -6.78 & \\
\hline 449 & 2234.18 & 2233.89 & 18.00 & 18.29 & -6.54 & -7.54 \\
\hline 450 & 2239.14 & & 17.50 & & -6.35 & \\
\hline 451 & 2244.11 & 2243.38 & 16.36 & 16.50 & -6.40 & -7.70 \\
\hline 452 & 2249.20 & & 15.51 & & -6.21 & \\
\hline 453 & 2254.29 & 2253.76 & 14.66 & 15.02 & -6.01 & -7.46 \\
\hline 454 & 2259.12 & & 14.77 & & -5.47 & \\
\hline 455 & 2264.43 & 2263.35 & 14.87 & 15.17 & -5.61 & -7.33 \\
\hline 456 & 2269.04 & & 14.85 & & -5.48 & \\
\hline 457 & 2273.80 & 2273.98 & 14.80 & 14.99 & -5.09 & -5.72 \\
\hline 458 & 2279.04 & & 14.83 & & -4.84 & \\
\hline 459 & 2283.59 & 2283.61 & 15.08 & 15.25 & -4.49 & -5.71 \\
\hline 460 & 2288.98 & & 14.41 & & -4.42 & \\
\hline 461 & 2294.52 & 2293.91 & 14.67 & 14.77 & -3.90 & -5.34 \\
\hline 462 & 2299.44 & & 15.23 & & -4.14 & \\
\hline 463 & 2303.51 & 2303.86 & 15.70 & 15.84 & -4.28 & -5.39 \\
\hline 464 & 2308.68 & & 16.11 & & -4.99 & \\
\hline 465 & 2313.85 & 2312.98 & 16.40 & 16.32 & -4.72 & -5.79 \\
\hline 466 & 2319.01 & & 16.83 & & -4.10 & \\
\hline 467 & 2324.24 & 2323.48 & 17.17 & 17.37 & -2.67 & -3.97 \\
\hline 468 & 2329.34 & & 17.29 & & -2.12 & \\
\hline 469 & 2334.22 & 2333.75 & 17.42 & 17.70 & -1.88 & -3.36 \\
\hline 470 & 2339.16 & & 19.67 & & -1.59 & \\
\hline 471 & 2343.25 & & 21.61 & & -2.12 & \\
\hline 472 & 2348.74 & & 22.76 & & -2.16 & \\
\hline 473 & 2352.91 & & 22.39 & & -1.92 & \\
\hline 474 & 2358.19 & & 21.87 & & -1.84 & \\
\hline 475 & 2362.91 & & 21.90 & & -1.55 & \\
\hline 476 & 2367.64 & & 19.92 & & -3.25 & \\
\hline 477 & 2372.77 & 2373.39 & 18.07 & 17.94 & -3.66 & -4.71 \\
\hline 478 & 2377.24 & & 19.08 & & -3.41 & \\
\hline 479 & 2382.71 & 2383.71 & 21.45 & 21.18 & -3.56 & -4.30 \\
\hline 480 & 2388.13 & & 18.12 & & -3.36 & \\
\hline
\end{tabular}

Appendix A, page 9 


\begin{tabular}{|c|c|c|c|c|c|c|}
\hline 481 & 2393.03 & 2393.77 & 18.25 & 18.41 & -3.88 & -4.55 \\
\hline 482 & 2398.21 & & 19.69 & & -3.75 & \\
\hline 483 & 2403.47 & 2403.66 & 20.31 & 20.42 & -3.94 & -4.63 \\
\hline 484 & 2408.40 & & 21.23 & & -3.40 & \\
\hline 485 & 2413.61 & 2413.22 & 21.07 & 21.90 & -3.02 & -4.43 \\
\hline 486 & 2418.20 & & 21.00 & & -3.22 & \\
\hline 487 & 2422.31 & 2422.10 & 19.04 & 19.20 & -4.00 & -4.88 \\
\hline 488 & 2427.06 & & 21.38 & & -3.54 & \\
\hline 489 & 2432.37 & 2432.15 & 22.57 & 22.60 & -3.40 & -4.33 \\
\hline 490 & 2437.19 & & 22.12 & & -3.55 & \\
\hline 491 & 2442.50 & 2443.95 & 19.38 & 19.52 & -4.10 & -5.13 \\
\hline 492 & 2447.29 & & 19.52 & & -3.95 & \\
\hline 493 & 2452.25 & 2454.24 & 22.56 & 22.20 & -2.83 & -4.25 \\
\hline 494 & 2457.10 & & 21.36 & & -2.79 & \\
\hline 495 & 2462.03 & 2465.06 & 20.89 & 21.15 & -2.66 & -3.55 \\
\hline 496 & 2467.17 & & 21.13 & & -2.59 & \\
\hline 497 & 2472.09 & 2474.70 & 21.17 & 21.38 & -2.52 & -3.59 \\
\hline 498 & 2477.22 & & 21.27 & & -2.47 & \\
\hline 499 & 2482.20 & 2482.91 & 22.38 & 23.00 & -2.46 & -3.23 \\
\hline 500 & 2486.70 & & 25.26 & & -2.31 & \\
\hline 501 & 2491.37 & & 26.94 & & -2.34 & \\
\hline 502 & 2496.24 & & 24.75 & & -2.44 & \\
\hline 503 & 2501.32 & & 24.13 & & -2.09 & \\
\hline 504 & 2506.40 & 2505.70 & 23.91 & 24.01 & -1.64 & -2.80 \\
\hline 505 & 2511.26 & & 23.88 & & -1.27 & \\
\hline 506 & 2516.38 & 2515.83 & 23.83 & 23.66 & -1.02 & -2.42 \\
\hline 507 & 2521.31 & & 23.86 & & -1.03 & \\
\hline 508 & 2526.33 & 2526.73 & 24.01 & 24.13 & -0.51 & -1.71 \\
\hline 509 & 2531.38 & & 24.14 & & -0.25 & \\
\hline 510 & 2536.27 & 2535.63 & 24.36 & 23.99 & -0.05 & -1.47 \\
\hline 511 & 2541.31 & & 24.23 & & -0.01 & \\
\hline 512 & 2546.23 & 2544.23 & 23.15 & 22.79 & -0.20 & -2.10 \\
\hline 513 & 2551.34 & & 22.25 & & -0.26 & \\
\hline 514 & 2556.33 & 2554.83 & 22.92 & 22.95 & -0.21 & -2.11 \\
\hline 515 & 2561.36 & & 23.29 & & -0.23 & \\
\hline 516 & 2566.43 & 2566.21 & 22.82 & 22.92 & -0.29 & -2.32 \\
\hline 517 & 2571.37 & & 24.32 & & -0.46 & \\
\hline 518 & 2576.19 & 2576.12 & 25.04 & 25.14 & -0.50 & -2.02 \\
\hline 519 & 2581.24 & & 25.07 & & -0.69 & \\
\hline 520 & 2586.21 & 2586.04 & 25.10 & 25.25 & -0.82 & -1.67 \\
\hline 521 & 2591.24 & & 25.25 & & -0.75 & \\
\hline 522 & 2596.14 & 2596.02 & 25.43 & 25.44 & -0.48 & -1.52 \\
\hline 523 & 2601.14 & & 26.30 & & -0.81 & \\
\hline 524 & 2606.14 & 2605.94 & 26.90 & 26.62 & -0.93 & -1.97 \\
\hline 525 & 2611.04 & & 27.62 & & -1.06 & \\
\hline 526 & 2616.03 & 2616.40 & 26.35 & 26.48 & -0.94 & -1.69 \\
\hline 527 & 2621.00 & & 26.57 & & -0.73 & \\
\hline 528 & 2625.98 & 2626.36 & 26.76 & 26.64 & -0.53 & -1.38 \\
\hline 529 & 2631.06 & & 26.67 & & -0.44 & \\
\hline 530 & 2636.90 & 2635.72 & 26.69 & 25.12 & -0.41 & -1.94 \\
\hline 531 & 2641.01 & & 26.66 & & -0.55 & \\
\hline 532 & 2646.91 & 2644.98 & 26.80 & 25.34 & -0.14 & -1.75 \\
\hline 533 & 2650.99 & & 27.04 & & -0.17 & \\
\hline 534 & 2655.93 & 2655.88 & 27.53 & 27.68 & -0.05 & -0.78 \\
\hline
\end{tabular}

Appendix A, page 10 


\begin{tabular}{|c|c|c|c|c|c|c|}
\hline 535 & 2660.92 & & 28.12 & & -0.07 & \\
\hline 536 & 2665.59 & 2665.72 & 29.38 & 26.86 & -0.40 & -1.33 \\
\hline 537 & 2670.71 & & 26.81 & & -0.58 & \\
\hline 538 & 2675.84 & & 26.92 & & -1.02 & \\
\hline 539 & 2680.96 & & 26.98 & & -1.39 & \\
\hline 540 & 2685.70 & & 28.27 & & -1.71 & \\
\hline 541 & 2690.68 & & 28.65 & & -2.14 & \\
\hline 542 & 2695.82 & 2695.22 & 29.17 & 28.94 & -2.85 & -3.32 \\
\hline 543 & 2700.20 & & 29.80 & & -2.94 & \\
\hline 544 & 2705.29 & 2705.79 & 30.31 & 30.23 & -3.21 & -3.71 \\
\hline 545 & 2710.23 & & 30.22 & & -3.32 & \\
\hline 546 & 2715.35 & 2715.57 & 29.80 & 29.79 & -3.44 & -3.89 \\
\hline 547 & 2720.42 & & 28.83 & & -3.30 & \\
\hline 548 & 2725.69 & & 29.30 & & -3.83 & \\
\hline 549 & 2730.40 & & 30.26 & & -4.20 & \\
\hline 550 & 2735.37 & 2736.26 & 32.23 & 33.26 & -4.42 & -5.45 \\
\hline 551 & 2740.19 & & 34.36 & & -4.64 & \\
\hline 552 & 2745.02 & 2745.25 & 36.46 & 36.54 & -5.05 & -5.78 \\
\hline 553 & 2750.07 & & 38.01 & & -5.33 & \\
\hline 554 & 2754.45 & 2755.63 & 39.37 & 39.87 & -5.46 & -6.20 \\
\hline 555 & 2759.17 & & 39.90 & & -6.01 & \\
\hline 556 & 2764.44 & 2765.18 & 39.97 & 39.77 & -6.04 & -6.45 \\
\hline 557 & 2769.22 & & 39.95 & & -6.24 & \\
\hline 558 & 2774.54 & 2775.52 & 40.92 & 40.51 & -6.21 & -7.11 \\
\hline 559 & 2779.33 & & 39.83 & & -7.13 & \\
\hline 560 & 2784.10 & & 40.08 & & -6.80 & \\
\hline 561 & 2789.15 & & 40.11 & & -7.17 & \\
\hline 562 & 2794.26 & 2794.24 & 40.25 & 39.72 & -7.29 & -8.28 \\
\hline 563 & 2799.36 & & 40.14 & & -7.95 & \\
\hline 564 & 2804.15 & 2805.63 & 39.50 & 39.36 & -7.38 & -8.15 \\
\hline 565 & 2809.17 & & 38.43 & & -7.88 & \\
\hline 566 & 2814.10 & 2814.74 & 37.40 & 37.73 & -8.17 & -8.48 \\
\hline 567 & 2819.15 & & 36.40 & & -8.42 & \\
\hline 568 & 2824.20 & 2824.97 & 36.89 & 37.44 & -8.19 & -8.71 \\
\hline 569 & 2829.14 & & 37.95 & & -8.84 & \\
\hline 570 & 2834.12 & 2835.26 & 38.46 & 38.87 & -9.20 & -10.01 \\
\hline 571 & 2839.18 & & 38.07 & & -9.17 & \\
\hline 572 & 2844.04 & 2845.10 & 37.64 & 37.91 & -9.02 & -9.73 \\
\hline 573 & 2849.27 & & 36.65 & & -9.20 & \\
\hline 574 & 2854.16 & 2854.67 & 36.76 & 37.60 & -9.62 & -10.36 \\
\hline 575 & 2859.16 & & 36.78 & & -9.68 & \\
\hline 576 & 2864.20 & 2865.07 & 36.80 & 37.62 & -9.70 & -10.69 \\
\hline 577 & 2869.25 & & 37.70 & & -9.91 & \\
\hline 578 & 2874.21 & 2875.26 & 38.53 & 39.55 & -9.91 & -10.82 \\
\hline 579 & 2879.10 & & 39.46 & & -10.23 & \\
\hline 580 & 2884.03 & 2885.45 & 39.92 & 40.87 & -10.21 & -10.92 \\
\hline 581 & 2889.10 & & 40.66 & & -10.18 & \\
\hline 582 & 2893.69 & 2895.04 & 40.63 & 41.62 & -10.88 & -11.93 \\
\hline 583 & 2898.93 & & 41.58 & & -10.68 & \\
\hline 584 & 2904.06 & 2905.46 & 41.35 & 41.95 & -11.17 & -11.80 \\
\hline 585 & 2908.94 & & 40.48 & & -10.98 & \\
\hline 586 & 2913.51 & 2914.85 & 39.23 & 40.17 & -11.24 & -12.05 \\
\hline 587 & 2917.55 & & 38.58 & & -10.64 & \\
\hline 588 & 2924.12 & 2924.80 & 37.07 & 37.99 & -9.38 & -10.41 \\
\hline
\end{tabular}

Appendix A, page 11 


\begin{tabular}{|c|c|c|c|c|c|c|}
\hline 589 & 2928.58 & & 35.91 & & -9.46 & \\
\hline 590 & 2933.72 & 2934.43 & 35.07 & 36.33 & -9.52 & -10.28 \\
\hline 591 & 2939.84 & & 37.61 & & -9.45 & \\
\hline 592 & 2944.41 & 2944.20 & 38.36 & 39.89 & -9.13 & -10.00 \\
\hline 593 & 2948.85 & & 38.62 & & -9.67 & \\
\hline 594 & 2953.96 & 2954.86 & 37.84 & 37.84 & -10.74 & -11.73 \\
\hline 595 & 2958.98 & & 38.34 & & -8.87 & \\
\hline 596 & 2964.15 & 2964.58 & 38.90 & 40.30 & -9.09 & -10.38 \\
\hline 597 & 2969.10 & & 37.23 & & -8.81 & \\
\hline 598 & 2974.14 & 2974.75 & 37.50 & 37.90 & -9.01 & -9.87 \\
\hline 599 & 2979.19 & & 38.03 & & -9.13 & \\
\hline 600 & 2983.91 & 2984.75 & 38.79 & 39.07 & -9.32 & -10.60 \\
\hline 601 & 2989.25 & & 38.34 & & -9.43 & \\
\hline 602 & 2994.19 & 2994.91 & 40.46 & 41.02 & -9.59 & -11.04 \\
\hline 603 & 2999.26 & & 41.67 & & -8.51 & \\
\hline 604 & 3004.12 & 3004.36 & 42.35 & 42.87 & -8.48 & -9.56 \\
\hline 605 & 3009.07 & & 43.28 & & -8.18 & \\
\hline 606 & 3014.05 & 3013.97 & 44.16 & 44.56 & -8.28 & -9.26 \\
\hline 607 & 3018.03 & & 44.78 & & -7.97 & \\
\hline 608 & 3023.43 & 3023.92 & 46.81 & 47.28 & -8.06 & -8.70 \\
\hline 609 & 3027.68 & & 48.26 & & -7.58 & \\
\hline 610 & 3033.11 & 3032.82 & 50.75 & 50.88 & -7.52 & -8.13 \\
\hline 611 & 3037.34 & & 52.76 & & -7.39 & \\
\hline 612 & 3041.61 & 3042.37 & 54.77 & 55.45 & -7.13 & -8.20 \\
\hline 613 & 3046.48 & & 56.84 & & -7.09 & \\
\hline 614 & 3052.19 & 3051.94 & 59.13 & 59.37 & -7.01 & -8.02 \\
\hline 615 & 3056.93 & & 60.70 & & -6.85 & \\
\hline 616 & 3061.64 & 3061.58 & 62.11 & 62.45 & -6.95 & -7.85 \\
\hline 617 & 3066.50 & & 63.31 & & -6.75 & \\
\hline 618 & 3071.17 & 3071.15 & 64.53 & 64.85 & -6.56 & -7.55 \\
\hline 619 & 3076.14 & & 65.99 & & -6.48 & \\
\hline 620 & 3081.10 & 3080.87 & 67.34 & 67.52 & -6.31 & -7.36 \\
\hline 621 & 3085.78 & & 68.28 & & -6.20 & \\
\hline 622 & 3090.83 & 3090.93 & 68.99 & 69.20 & -5.98 & -7.01 \\
\hline 623 & 3095.56 & & 69.75 & & -5.94 & \\
\hline 624 & 3100.85 & 3100.85 & 70.42 & 70.56 & -5.68 & -6.59 \\
\hline 625 & 3105.86 & & 71.07 & & -5.57 & \\
\hline 626 & 3110.70 & 3110.59 & 71.65 & 71.78 & -5.30 & -6.22 \\
\hline 627 & 3115.90 & & 72.14 & & -4.98 & \\
\hline 628 & 3120.77 & 3120.52 & 72.70 & 72.94 & -4.78 & -5.77 \\
\hline 629 & 3125.84 & & 72.89 & & -4.37 & \\
\hline 630 & 3130.74 & 3130.15 & 73.07 & 73.25 & -4.10 & -4.75 \\
\hline 631 & 3135.78 & & 73.32 & & -3.83 & \\
\hline 632 & 3140.68 & 3141.71 & 73.43 & 73.63 & -3.56 & -4.34 \\
\hline 633 & 3145.72 & & 73.58 & & -3.37 & \\
\hline 634 & 3150.66 & 3150.54 & 73.69 & 74.00 & -3.18 & -4.09 \\
\hline 635 & 3155.78 & & 73.78 & & -3.04 & \\
\hline 636 & 3160.80 & 3160.48 & 73.73 & 74.13 & -2.68 & -3.52 \\
\hline 637 & 3165.73 & & 73.45 & & -2.57 & \\
\hline 638 & 3170.76 & 3171.17 & 73.27 & 73.62 & -2.39 & -3.25 \\
\hline 639 & 3175.72 & & 73.15 & & -2.25 & \\
\hline 640 & 3180.66 & 3180.43 & 73.01 & 73.36 & -2.09 & -2.80 \\
\hline 641 & 3185.82 & & 72.95 & & -1.93 & \\
\hline 642 & 3190.69 & 3189.94 & 73.16 & 73.47 & -1.94 & -2.60 \\
\hline
\end{tabular}

Appendix A, page 12 


\begin{tabular}{|c|c|c|c|c|c|c|}
\hline 643 & 3195.76 & & 73.12 & & -1.80 & \\
\hline 644 & 3200.67 & 3200.97 & 72.58 & 72.88 & -1.78 & -2.46 \\
\hline 645 & 3206.07 & & 71.66 & & -1.03 & \\
\hline 646 & 3210.72 & 3210.71 & 73.82 & 74.24 & -1.20 & -2.03 \\
\hline 647 & 3215.65 & & 75.20 & & -0.89 & \\
\hline 648 & 3220.60 & 3220.46 & 77.29 & 77.60 & -0.38 & -1.20 \\
\hline 649 & 3224.94 & & 79.46 & & -0.02 & \\
\hline 650 & 3229.39 & 3229.28 & 82.88 & 83.12 & 0.40 & -0.49 \\
\hline 651 & 3233.95 & & 85.08 & & 0.61 & \\
\hline 652 & 3238.57 & 3238.51 & 86.60 & 86.65 & 1.13 & 0.23 \\
\hline 653 & 3243.19 & & 88.66 & & 0.88 & \\
\hline 654 & 3247.97 & 3247.57 & 89.67 & 89.70 & 0.86 & -0.05 \\
\hline 655 & 3252.53 & & 91.47 & & 1.02 & \\
\hline 656 & 3257.00 & 3256.93 & 94.22 & 94.30 & 1.17 & 0.33 \\
\hline 657 & 3261.41 & & 96.83 & & 1.10 & \\
\hline 658 & 3265.87 & 3265.45 & 98.92 & 98.77 & 0.88 & 0.05 \\
\hline 659 & 3270.40 & & 101.06 & & 0.98 & \\
\hline 660 & 3274.93 & 3274.57 & 103.18 & 102.95 & 1.10 & 0.21 \\
\hline 661 & 3279.55 & & 105.15 & & 1.19 & \\
\hline 662 & 3284.02 & 3283.69 & 107.14 & 107.12 & 1.04 & 0.13 \\
\hline 663 & 3288.60 & & 108.92 & & 1.06 & \\
\hline 664 & 3293.19 & 3292.81 & 110.71 & 110.80 & 1.07 & 0.07 \\
\hline 665 & 3297.91 & & 112.38 & & 0.90 & \\
\hline 666 & 3302.60 & 3302.43 & 113.90 & 113.98 & 1.09 & 0.02 \\
\hline 667 & 3307.39 & & 115.29 & & 1.14 & \\
\hline 668 & 3312.26 & 3312.05 & 116.17 & 116.25 & 1.09 & -0.04 \\
\hline 669 & 3318.89 & & 117.11 & & 0.68 & \\
\hline 670 & 3323.80 & 3323.77 & 117.61 & 117.83 & 0.79 & -0.30 \\
\hline 671 & 3328.87 & & 117.88 & & 0.23 & \\
\hline 672 & 3333.56 & 3333.27 & 117.75 & 118.04 & 0.20 & -0.46 \\
\hline 673 & 3338.75 & & 116.85 & & 0.17 & \\
\hline 674 & 3343.43 & 3343.38 & 115.79 & 116.14 & 0.44 & -0.62 \\
\hline 675 & 3348.46 & & 114.74 & & 0.28 & \\
\hline 676 & 3353.44 & 3353.48 & 113.81 & 114.25 & 0.35 & -0.78 \\
\hline 677 & 3358.33 & & 113.24 & & 0.29 & \\
\hline 678 & 3363.41 & 3363.20 & 112.79 & 113.11 & 1.27 & 0.38 \\
\hline 679 & 3368.61 & & 112.96 & & 0.67 & \\
\hline 680 & 3373.72 & 3373.22 & 112.94 & 113.31 & 0.70 & -0.32 \\
\hline 681 & 3378.70 & & 112.91 & & 0.90 & \\
\hline 682 & 3383.43 & 3384.00 & 112.84 & 113.06 & 1.11 & 0.29 \\
\hline 683 & 3388.39 & & 112.59 & & 1.65 & \\
\hline 684 & 3393.37 & 3394.23 & 112.34 & 112.39 & 1.98 & 1.05 \\
\hline 685 & 3398.28 & & 111.52 & & 2.06 & \\
\hline 686 & 3403.03 & 3402.95 & 110.21 & 110.21 & 2.05 & 0.99 \\
\hline 687 & 3407.77 & & 108.73 & & 2.06 & \\
\hline 688 & 3412.85 & 3413.04 & 107.67 & 107.78 & 2.13 & 1.24 \\
\hline 689 & 3417.82 & & 107.13 & & 1.92 & \\
\hline 690 & 3422.75 & 3422.46 & 106.62 & 106.83 & 1.81 & 1.00 \\
\hline 691 & 3427.86 & & 105.98 & & 2.24 & \\
\hline 692 & 3432.77 & 3433.17 & 105.22 & 105.24 & 1.71 & 1.04 \\
\hline 693 & 3437.78 & & 104.27 & & 2.38 & \\
\hline 694 & 3442.60 & 3442.71 & 103.42 & 103.54 & 2.84 & 1.71 \\
\hline 695 & 3447.47 & & 102.53 & & 2.80 & \\
\hline 696 & 3452.39 & 3452.98 & 101.85 & 101.88 & 3.05 & 2.32 \\
\hline
\end{tabular}

Appendix A, page 13 


\begin{tabular}{|c|c|c|c|c|c|c|}
\hline 697 & 3457.23 & & 101.03 & & 3.29 & \\
\hline 698 & 3462.29 & 3461.67 & 100.18 & 100.52 & 3.41 & 2.57 \\
\hline 699 & 3467.31 & & 99.08 & & 3.72 & \\
\hline 700 & 3472.55 & 3471.57 & 97.87 & 98.31 & 3.91 & 3.06 \\
\hline 701 & 3477.08 & & 96.70 & & 4.02 & \\
\hline 702 & 3482.04 & 3482.16 & 95.71 & 95.91 & 4.79 & 3.62 \\
\hline 703 & 3487.13 & & 95.88 & & 4.88 & \\
\hline 704 & 3492.12 & 3492.25 & 97.72 & 97.79 & 5.32 & 4.22 \\
\hline 705 & 3496.87 & & 99.84 & & 5.34 & \\
\hline 706 & 3501.48 & 3501.58 & 101.39 & 101.55 & 5.55 & 4.76 \\
\hline 707 & 3506.00 & & 101.61 & & 5.00 & \\
\hline 708 & 3511.00 & 3511.65 & 101.61 & 101.53 & 5.00 & 4.23 \\
\hline 709 & 3516.52 & & 102.17 & & 4.99 & \\
\hline 710 & 3521.76 & 3521.71 & 101.49 & 101.50 & 4.59 & 3.69 \\
\hline 711 & 3526.64 & & 100.56 & & 4.73 & \\
\hline 712 & 3531.60 & 3531.56 & 99.72 & 99.72 & 5.15 & 4.24 \\
\hline 713 & 3536.52 & & 98.97 & & 5.56 & \\
\hline 714 & 3541.38 & 3541.38 & 98.37 & 98.39 & 5.93 & 4.99 \\
\hline 715 & 3546.47 & & 97.96 & & 6.40 & \\
\hline 716 & 3551.44 & 3551.36 & 97.67 & 97.65 & 6.89 & 5.85 \\
\hline 717 & 3556.04 & & 97.31 & & 7.26 & \\
\hline 718 & 3561.75 & 3562.31 & 97.34 & 97.41 & 7.60 & 6.64 \\
\hline 719 & 3566.30 & & 97.25 & & 7.50 & \\
\hline 720 & 3571.42 & 3572.06 & 96.86 & 96.90 & 7.58 & 6.68 \\
\hline 721 & 3576.30 & & 96.43 & & 7.59 & \\
\hline 722 & 3581.33 & 3581.76 & 95.80 & 95.80 & 7.74 & 6.84 \\
\hline 723 & 3586.26 & & 95.31 & & 7.84 & \\
\hline 724 & 3591.17 & 3591.60 & 94.77 & 94.77 & 7.89 & 6.99 \\
\hline 725 & 3596.27 & & 94.12 & & 7.55 & \\
\hline 726 & 3601.11 & 3601.54 & 93.46 & 93.46 & 7.31 & 6.41 \\
\hline 727 & 3606.07 & & 92.73 & & 7.26 & \\
\hline 728 & 3610.85 & 3611.28 & 91.62 & 91.62 & 6.93 & 6.03 \\
\hline 729 & 3615.97 & & 90.61 & & 6.74 & \\
\hline 730 & 3621.57 & 3621.09 & 89.87 & 89.97 & 7.12 & 5.95 \\
\hline 731 & 3625.26 & & 89.58 & & 6.59 & \\
\hline 732 & 3630.13 & 3630.39 & 89.44 & 89.32 & 7.00 & 5.62 \\
\hline 733 & 3635.04 & & 88.68 & & 6.42 & \\
\hline 734 & 3640.45 & 3640.78 & 87.74 & 87.79 & 5.96 & 4.16 \\
\hline 735 & 3644.91 & & 86.78 & & 6.20 & \\
\hline 736 & 3649.79 & 3650.00 & 86.01 & 86.11 & 6.05 & 5.00 \\
\hline 737 & 3655.01 & & 84.75 & & 6.18 & \\
\hline 738 & 3659.91 & 3660.34 & 83.35 & 83.35 & 6.51 & 5.61 \\
\hline 739 & 3664.31 & & 82.11 & & 6.15 & \\
\hline 740 & 3669.18 & 3669.61 & 80.59 & 80.59 & 6.42 & 5.52 \\
\hline 741 & 3674.05 & & 78.84 & & 6.23 & \\
\hline 742 & 3678.33 & 3678.76 & 77.20 & 77.20 & 6.24 & 5.34 \\
\hline 743 & 3683.25 & & 75.32 & & 6.32 & \\
\hline 744 & 3688.29 & 3688.72 & 73.72 & 73.72 & 6.53 & 5.63 \\
\hline 745 & 3693.15 & & 72.37 & & 6.50 & \\
\hline 746 & 3697.95 & 3698.38 & 71.21 & 71.21 & 6.83 & 5.93 \\
\hline 747 & 3702.75 & & 70.19 & & 6.65 & \\
\hline 748 & 3707.65 & 3708.08 & 69.19 & 69.19 & 6.66 & 5.76 \\
\hline 749 & 3712.71 & & 68.22 & & 6.97 & \\
\hline 750 & 3717.74 & & 67.04 & & 7.12 & \\
\hline
\end{tabular}

Appendix A, page 14 


\begin{tabular}{|c|c|c|c|c|c|c|}
\hline 751 & 3722.37 & & 65.77 & & 6.92 & \\
\hline 752 & 3727.18 & 3727.75 & 64.04 & 64.19 & 7.14 & 6.00 \\
\hline 753 & 3732.00 & & 65.61 & & 7.00 & \\
\hline 754 & 3737.51 & 3738.14 & 65.82 & 65.62 & 7.22 & 6.05 \\
\hline 755 & 3742.37 & & 67.03 & & 7.16 & \\
\hline 756 & 3747.45 & 3747.88 & 67.87 & 67.87 & 7.09 & 6.19 \\
\hline 757 & 3752.17 & & 68.20 & & 7.52 & \\
\hline 758 & 3757.25 & 3757.99 & 67.55 & 67.59 & 7.44 & 6.35 \\
\hline 759 & 3761.13 & & 67.06 & & 7.66 & \\
\hline 760 & 3767.24 & 3767.80 & 65.89 & 65.91 & 7.63 & 6.60 \\
\hline 761 & 3772.22 & & 65.07 & & 7.87 & \\
\hline 762 & 3777.14 & 3777.71 & 64.27 & 64.22 & 7.88 & 6.74 \\
\hline 763 & 3782.00 & & 63.50 & & 7.65 & \\
\hline 764 & 3787.09 & 3787.57 & 63.27 & 63.11 & 8.04 & 6.85 \\
\hline 765 & 3792.38 & & 63.33 & & 8.23 & \\
\hline 766 & 3797.34 & & 63.57 & & 8.37 & \\
\hline 767 & 3801.96 & & 64.08 & & 8.22 & \\
\hline 768 & 3806.72 & 3807.50 & 64.65 & 64.69 & 8.31 & 7.28 \\
\hline 769 & 3811.80 & & 65.00 & & 8.38 & \\
\hline 770 & 3816.89 & 3817.53 & 65.39 & 65.35 & 8.64 & 7.49 \\
\hline 771 & 3821.99 & & 65.36 & & 8.85 & \\
\hline 772 & 3826.97 & 3827.70 & 65.08 & 65.02 & 9.12 & 7.53 \\
\hline 773 & 3831.43 & & 64.29 & & 9.46 & \\
\hline 774 & 3836.79 & 3837.43 & 63.60 & 63.61 & 9.08 & 8.10 \\
\hline 775 & 3841.85 & & 62.83 & & 9.33 & \\
\hline 776 & 3846.31 & 3847.27 & 61.99 & 61.99 & 9.93 & 8.27 \\
\hline 777 & 3851.55 & & 60.91 & & 9.75 & \\
\hline 778 & 3856.51 & 3857.00 & 59.99 & 59.96 & 9.60 & 8.26 \\
\hline 779 & 3861.42 & & 58.70 & & 9.80 & \\
\hline 780 & 3866.22 & 3866.87 & 57.52 & 57.58 & 9.63 & 8.55 \\
\hline 781 & 3870.87 & & 56.40 & & 10.01 & \\
\hline 782 & 3875.69 & 3876.35 & 55.17 & 55.26 & 10.12 & 9.15 \\
\hline 783 & 3880.75 & & 54.01 & & 10.19 & \\
\hline 784 & 3884.76 & 3886.18 & 52.87 & 52.75 & 10.27 & 9.26 \\
\hline 785 & 3890.11 & & 51.41 & & 10.08 & \\
\hline 786 & 3895.14 & & 50.22 & & 10.48 & \\
\hline 787 & 3899.97 & & 49.05 & & 10.79 & \\
\hline 788 & 3904.96 & 3905.62 & 48.46 & 48.35 & 10.80 & 9.37 \\
\hline 789 & 3910.06 & & 47.57 & & 10.93 & \\
\hline 790 & 3915.01 & & 47.52 & & 11.06 & \\
\hline 791 & 3919.93 & & 47.18 & & 11.10 & \\
\hline 792 & 3924.93 & 3925.47 & 46.87 & 46.74 & 11.31 & 9.86 \\
\hline 793 & 3929.83 & & 46.62 & & 11.42 & \\
\hline 794 & 3934.52 & 3935.55 & 46.03 & 46.03 & 11.31 & 10.31 \\
\hline 795 & 3939.62 & & 45.23 & & 11.67 & \\
\hline 796 & 3944.72 & 3945.26 & 44.44 & 44.60 & 12.04 & 11.21 \\
\hline 797 & 3949.82 & & 43.64 & & 12.40 & \\
\hline 798 & 3954.45 & 3955.07 & 42.95 & 42.95 & 12.71 & 11.73 \\
\hline 799 & 3959.43 & & 42.12 & & 12.74 & \\
\hline 800 & 3964.14 & 3964.79 & 41.46 & 41.57 & 13.05 & 12.20 \\
\hline 801 & 3969.72 & & 40.49 & & 13.49 & \\
\hline 802 & 3974.24 & 3974.96 & 39.41 & 39.43 & 13.52 & 12.47 \\
\hline 803 & 3978.73 & & 38.51 & & 13.78 & \\
\hline 804 & 3983.61 & 3984.24 & 37.23 & 37.30 & 13.70 & 12.80 \\
\hline
\end{tabular}

Appendix A, page 15 


\begin{tabular}{|c|c|c|c|c|c|c|}
\hline 805 & 3988.73 & & 35.57 & & 14.03 & \\
\hline 806 & 3993.05 & 3993.83 & 34.37 & 34.16 & 13.76 & 12.30 \\
\hline 807 & 3998.49 & & 32.65 & & 13.00 & \\
\hline 808 & 4002.84 & 4003.67 & 31.56 & 31.48 & 14.14 & 13.36 \\
\hline 809 & 4007.95 & & 30.19 & & 14.42 & \\
\hline 810 & 4012.54 & 4013.24 & 28.72 & 28.77 & 14.63 & 13.63 \\
\hline 811 & 4017.42 & & 26.77 & & 14.58 & \\
\hline 812 & 4022.12 & 4022.34 & 24.96 & 25.33 & 14.72 & 13.77 \\
\hline 813 & 4026.57 & & 23.27 & & 14.50 & \\
\hline 814 & 4031.77 & 4032.47 & 21.52 & 21.51 & 14.80 & 13.80 \\
\hline 815 & 4036.33 & & 19.85 & & 14.85 & \\
\hline 816 & 4040.78 & 4041.39 & 18.19 & 18.35 & 14.93 & 13.95 \\
\hline 817 & 4045.50 & & 16.00 & & 14.30 & \\
\hline 818 & 4050.65 & 4050.99 & 18.65 & 18.51 & 14.39 & 13.01 \\
\hline 819 & 4056.09 & & 20.67 & & 13.92 & \\
\hline 820 & 4060.13 & 4060.89 & 22.40 & 22.46 & 13.78 & 12.73 \\
\hline 821 & 4064.81 & & 23.71 & & 14.03 & \\
\hline 822 & 4069.66 & 4070.66 & 23.51 & 23.49 & 13.58 & 12.35 \\
\hline 823 & 4075.03 & & 23.71 & & 13.97 & \\
\hline 824 & 4079.44 & 4079.81 & 20.81 & 20.51 & 13.10 & 11.97 \\
\hline 825 & 4084.03 & & 19.47 & & 13.03 & \\
\hline 826 & 4088.74 & 4089.50 & 18.74 & 18.76 & 12.84 & 11.90 \\
\hline 827 & 4093.98 & & 18.13 & & 12.64 & \\
\hline 828 & 4099.08 & 4099.75 & 17.89 & 17.92 & 12.47 & 11.53 \\
\hline 829 & 4103.89 & & 18.15 & & 12.24 & \\
\hline 830 & 4108.51 & 4109.40 & 18.21 & 18.14 & 12.09 & 10.43 \\
\hline 831 & 4113.74 & & 18.30 & & 11.29 & \\
\hline 832 & 4118.81 & 4119.51 & 18.49 & 18.58 & 11.02 & 9.92 \\
\hline 833 & 4123.57 & & 18.67 & & 10.85 & \\
\hline 834 & 4128.75 & & 18.68 & & 10.98 & \\
\hline 835 & 4134.36 & & 18.93 & & 10.85 & \\
\hline 836 & 4138.77 & 4139.52 & 19.31 & 19.49 & 10.57 & 9.48 \\
\hline 837 & 4143.74 & & 19.54 & & 11.13 & \\
\hline 838 & 4148.79 & 4149.46 & 19.68 & 19.81 & 11.21 & 10.09 \\
\hline 839 & 4153.78 & & 19.76 & & 11.29 & \\
\hline 840 & 4158.85 & 4159.42 & 19.16 & 19.33 & 11.32 & 10.36 \\
\hline 841 & 4163.73 & & 20.08 & & 11.24 & \\
\hline 842 & 4168.85 & 4169.28 & 21.20 & 21.47 & 11.53 & 10.58 \\
\hline 843 & 4173.54 & & 21.53 & & 11.48 & \\
\hline 844 & 4178.27 & 4179.17 & 22.08 & 22.67 & 11.96 & 10.73 \\
\hline 845 & 4183.93 & & 23.78 & & 11.58 & \\
\hline 846 & 4189.07 & 4189.50 & 25.12 & 25.35 & 11.65 & 10.71 \\
\hline 847 & 4193.68 & & 26.02 & & 11.61 & \\
\hline 848 & 4198.46 & 4199.09 & 26.77 & 27.18 & 11.32 & 10.32 \\
\hline 849 & 4203.45 & & 27.57 & & 11.46 & \\
\hline 850 & 4208.18 & 4209.03 & 27.71 & 28.05 & 11.49 & 10.60 \\
\hline 851 & 4213.25 & & 27.83 & & 10.73 & \\
\hline 852 & 4218.35 & 4219.19 & 27.24 & 27.83 & 10.28 & 9.19 \\
\hline 853 & 4223.00 & & 26.88 & & 10.38 & \\
\hline 854 & 4228.17 & 4228.85 & 27.22 & 26.84 & 10.25 & 8.70 \\
\hline 855 & 4232.92 & & 28.75 & & 10.60 & \\
\hline 856 & 4237.76 & 4238.44 & 30.27 & 30.10 & 10.63 & 9.95 \\
\hline 857 & 4242.57 & & 31.22 & & 10.89 & \\
\hline 858 & 4247.78 & 4248.52 & 32.61 & 32.33 & 11.59 & 10.50 \\
\hline
\end{tabular}

\section{Appendix A, page 16}




\begin{tabular}{|c|c|c|c|c|c|c|}
\hline 859 & 4252.50 & & 33.71 & & 11.88 & \\
\hline 860 & 4257.14 & 4257.92 & 35.02 & 34.93 & 11.97 & 10.79 \\
\hline 861 & 4262.30 & & 36.54 & & 12.04 & \\
\hline 862 & 4267.10 & 4267.81 & 37.63 & 37.64 & 11.84 & 10.47 \\
\hline 863 & 4272.15 & & 38.73 & & 11.75 & \\
\hline 864 & 4276.83 & 4277.60 & 39.20 & 39.30 & 11.68 & 10.69 \\
\hline 865 & 4281.84 & & 39.70 & & 11.74 & \\
\hline 866 & 4286.70 & 4287.08 & 40.26 & 40.43 & 12.06 & 10.76 \\
\hline 867 & 4291.69 & & 40.77 & & 12.36 & \\
\hline 868 & 4296.67 & 4297.04 & 41.29 & 41.50 & 12.65 & 11.63 \\
\hline 869 & 4301.64 & & 41.66 & & 12.86 & \\
\hline 870 & 4306.61 & 4307.08 & 42.02 & 42.27 & 13.06 & 12.07 \\
\hline 871 & 4311.53 & & 42.29 & & 13.38 & \\
\hline 872 & 4316.45 & 4317.29 & 42.57 & 42.78 & 13.69 & 12.72 \\
\hline 873 & 4321.60 & & 42.74 & & 14.03 & \\
\hline 874 & 4326.76 & 4327.37 & 42.91 & 43.20 & 14.38 & 13.08 \\
\hline 875 & 4331.67 & & 42.93 & & 14.66 & \\
\hline 876 & 4336.58 & 4337.40 & 42.94 & 43.21 & 14.93 & 13.65 \\
\hline 877 & 4341.59 & & 42.92 & & 15.17 & \\
\hline 878 & 4346.60 & 4347.35 & 42.89 & 43.20 & 15.41 & 14.26 \\
\hline 879 & 4351.60 & & 42.12 & & 15.76 & \\
\hline 880 & 4356.60 & 4357.42 & 41.36 & 42.55 & 16.10 & 14.87 \\
\hline 881 & 4361.55 & & 40.68 & & 16.22 & \\
\hline 882 & 4366.49 & 4367.32 & 40.00 & 40.66 & 16.33 & 15.10 \\
\hline 883 & 4371.64 & & 39.60 & & 16.63 & \\
\hline 884 & 4376.79 & 4377.27 & 39.21 & 39.71 & 16.93 & 15.82 \\
\hline 885 & 4381.59 & & 38.57 & & 17.07 & \\
\hline 886 & 4386.40 & 4386.96 & 37.93 & 38.52 & 17.21 & 15.81 \\
\hline 887 & 4391.29 & & 37.62 & & 17.38 & \\
\hline 888 & 4396.19 & 4396.76 & 37.32 & 37.63 & 17.55 & 16.28 \\
\hline 889 & 4401.08 & & 36.81 & & 17.76 & \\
\hline 890 & 4405.98 & 4406.60 & 36.30 & 36.64 & 17.98 & 16.71 \\
\hline 891 & 4410.99 & & 35.77 & & 18.11 & \\
\hline 892 & 4416.00 & 4416.66 & 35.25 & 35.60 & 18.24 & 17.03 \\
\hline 893 & 4420.91 & & 34.45 & & 18.31 & \\
\hline 894 & 4425.81 & 4426.57 & 33.64 & 33.92 & 18.39 & 17.00 \\
\hline 895 & 4430.94 & & 33.19 & & 18.65 & \\
\hline 896 & 4436.06 & 4436.94 & 32.73 & 33.01 & 18.91 & 17.66 \\
\hline 897 & 4440.93 & & 32.85 & & 18.89 & \\
\hline 898 & 4445.79 & 4446.70 & 32.97 & 33.30 & 18.88 & 17.81 \\
\hline 899 & 4450.95 & & 33.03 & & 19.09 & \\
\hline 900 & 4456.11 & 4456.70 & 33.09 & 33.50 & 19.31 & 18.20 \\
\hline 901 & 4461.16 & & 32.71 & & 19.65 & \\
\hline 902 & 4466.20 & 4466.92 & 32.34 & 32.61 & 19.99 & 18.71 \\
\hline 903 & 4471.11 & & 33.11 & & 20.22 & \\
\hline 904 & 4476.02 & 4476.77 & 33.88 & 34.04 & 20.45 & 19.42 \\
\hline 905 & 4481.07 & & 34.14 & & 20.71 & \\
\hline 906 & 4486.12 & 4486.85 & 34.39 & 34.62 & 20.97 & 19.69 \\
\hline 907 & 4490.98 & & 34.85 & & 21.20 & \\
\hline 908 & 4495.83 & 4496.80 & 35.31 & 35.48 & 21.43 & 20.28 \\
\hline 909 & 4500.84 & & 35.79 & & 21.60 & \\
\hline 910 & 4505.85 & 4506.55 & 36.27 & 36.40 & 21.76 & 20.86 \\
\hline 911 & 4510.77 & & 36.85 & & 21.99 & \\
\hline 912 & 4515.68 & 4516.09 & 37.44 & 37.34 & 22.23 & 20.85 \\
\hline
\end{tabular}

Appendix A, page 17 


\begin{tabular}{|c|c|c|c|c|c|c|}
\hline 913 & 4520.73 & & 38.34 & & 22.43 & \\
\hline 914 & 4525.78 & 4526.17 & 39.24 & 39.07 & 22.64 & 21.36 \\
\hline 915 & 4530.77 & & 39.16 & & 22.84 & \\
\hline 916 & 4535.76 & 4536.57 & 39.08 & 38.77 & 23.05 & 21.60 \\
\hline 917 & 4540.80 & & 37.43 & & 23.20 & \\
\hline 918 & 4545.84 & 4546.28 & 35.79 & 35.95 & 23.35 & 22.50 \\
\hline 919 & 4550.92 & & 36.24 & & 23.87 & \\
\hline 920 & 4555.99 & 4556.51 & 36.69 & 36.93 & 24.39 & 23.15 \\
\hline 921 & 4560.92 & & 37.45 & & 24.65 & \\
\hline 922 & 4565.85 & 4566.61 & 38.20 & 38.56 & 24.92 & 23.90 \\
\hline 923 & 4570.54 & & 39.32 & & 25.27 & \\
\hline 924 & 4575.22 & 4576.09 & 40.44 & 40.85 & 25.62 & 24.54 \\
\hline 925 & 4580.24 & & 41.17 & & 25.74 & \\
\hline 926 & 4585.26 & 4585.82 & 41.90 & 42.34 & 25.86 & 24.86 \\
\hline 927 & 4590.30 & & 42.21 & & 25.98 & \\
\hline 928 & 4595.34 & 4595.78 & 42.53 & 42.96 & 26.10 & 24.97 \\
\hline 929 & 4600.33 & & 42.63 & & 26.14 & \\
\hline 930 & 4605.32 & 4605.95 & 42.73 & 43.10 & 26.17 & 25.06 \\
\hline 931 & 4610.37 & & 42.88 & & 26.19 & \\
\hline 932 & 4615.42 & 4615.86 & 43.04 & 43.49 & 26.22 & 25.08 \\
\hline 933 & 4620.35 & & 43.31 & & 26.17 & \\
\hline 934 & 4625.28 & 4625.69 & 43.58 & 43.83 & 26.12 & 25.00 \\
\hline 935 & 4630.24 & & 44.86 & & 26.15 & \\
\hline 936 & 4635.21 & 4635.80 & 46.14 & 46.57 & 26.17 & 24.98 \\
\hline 937 & 4640.15 & & 47.70 & & 26.12 & \\
\hline 938 & 4645.08 & 4645.99 & 49.27 & 49.77 & 26.08 & 24.87 \\
\hline 939 & 4649.81 & & 50.62 & & 26.35 & \\
\hline 940 & 4654.54 & 4655.04 & 51.98 & 52.51 & 26.61 & 25.41 \\
\hline 941 & 4659.28 & & 53.52 & & 26.66 & \\
\hline 942 & 4664.03 & 4664.72 & 55.07 & 55.53 & 26.70 & 25.66 \\
\hline 943 & 4668.82 & & 56.30 & & 26.79 & \\
\hline 944 & 4673.62 & 4674.43 & 57.53 & 58.04 & 26.88 & 25.61 \\
\hline 945 & 4678.61 & & 58.47 & & 27.14 & \\
\hline 946 & 4683.61 & 4684.15 & 59.42 & 59.75 & 27.41 & 26.26 \\
\hline 947 & 4688.54 & & 59.76 & & 27.48 & \\
\hline 948 & 4693.47 & 4693.18 & 60.10 & 60.61 & 27.54 & 26.57 \\
\hline 949 & 4698.45 & & 60.22 & & 27.62 & \\
\hline 950 & 4703.43 & 4704.06 & 60.33 & 61.03 & 27.71 & 26.70 \\
\hline 951 & 4708.18 & & 59.96 & & 27.64 & \\
\hline 952 & 4712.92 & 4713.16 & 59.59 & 60.28 & 27.58 & 26.84 \\
\hline 953 & 4718.17 & & 59.29 & & 27.52 & \\
\hline 954 & 4723.41 & 4724.10 & 58.99 & 59.59 & 27.46 & 26.18 \\
\hline 955 & 4728.36 & & 59.35 & & 27.53 & \\
\hline 956 & 4733.31 & 4733.96 & 59.71 & 60.43 & 27.60 & 26.49 \\
\hline 957 & 4738.38 & & 59.86 & & 27.76 & \\
\hline 958 & 4743.44 & 4744.10 & 60.02 & 60.64 & 27.92 & 26.78 \\
\hline 959 & 4748.54 & & 60.89 & & 28.16 & \\
\hline 960 & 4753.64 & 4753.73 & 61.77 & 62.45 & 28.40 & 27.31 \\
\hline 961 & 4758.46 & & 62.62 & & 28.36 & \\
\hline 962 & 4763.27 & 4764.28 & 63.47 & 64.20 & 28.33 & 27.16 \\
\hline 963 & 4768.53 & & 65.19 & & 28.03 & \\
\hline 964 & 4773.80 & 4774.59 & 66.91 & 67.39 & 27.73 & 26.68 \\
\hline 965 & 4778.20 & & 67.94 & & 28.48 & \\
\hline 966 & 4782.61 & 4783.24 & 68.97 & 69.46 & 29.23 & 28.20 \\
\hline
\end{tabular}

Appendix A, page 18 


\begin{tabular}{|c|c|c|c|c|c|c|}
\hline 967 & 4787.52 & & 69.78 & & 29.38 & \\
\hline 968 & 4792.43 & 4792.71 & 70.58 & 71.16 & 29.52 & 28.22 \\
\hline 969 & 4797.44 & & 70.37 & & 29.60 & \\
\hline 970 & 4802.44 & 4802.69 & 70.15 & 70.79 & 29.67 & 28.39 \\
\hline 971 & 4807.44 & & 70.23 & & 29.73 & \\
\hline 972 & 4812.43 & 4812.65 & 70.31 & 70.89 & 29.80 & 28.52 \\
\hline 973 & 4817.45 & & 70.40 & & 29.47 & \\
\hline 974 & 4822.47 & 4823.20 & 70.49 & 71.34 & 29.15 & 28.03 \\
\hline 975 & 4827.63 & & 70.70 & & 29.44 & \\
\hline 976 & 4832.79 & 4833.27 & 70.91 & 71.44 & 29.74 & 28.73 \\
\hline 977 & 4837.84 & & 70.75 & & 29.92 & \\
\hline 978 & 4842.90 & 4843.25 & 70.59 & 71.09 & 30.10 & 28.96 \\
\hline 979 & 4847.77 & & 70.51 & & 29.98 & \\
\hline 980 & 4852.64 & & 70.42 & & 29.85 & \\
\hline 981 & 4857.58 & & 70.11 & & 29.86 & \\
\hline 982 & 4862.53 & & 69.80 & & 29.88 & \\
\hline 983 & 4867.49 & & 68.72 & & 29.85 & \\
\hline 984 & 4872.45 & & 67.64 & & 29.82 & \\
\hline 985 & 4878.10 & & 65.83 & & 29.44 & \\
\hline 986 & 4883.75 & & 64.01 & & 29.07 & \\
\hline 987 & 4888.75 & & 62.01 & & 29.07 & \\
\hline 988 & 4893.75 & & 60.01 & & 29.07 & \\
\hline
\end{tabular}

